\title{
Instrumento para evaluar competencias matemáticas y científicas del alumnado que inicia Educación Primaria, mediante juegos
}

\author{
María José Espigares-Gámez ${ }^{1}$ \\ mery46@correo.ugr.es \\ https://orcid.org/0000-0002-0330-4507 \\ Alicia Fernández-Oliveras ${ }^{1}$ \\ alilia@ugr.es \\ https://orcid.org/0000-0001-5965-3389 \\ María Luisa Oliveras Contreras ${ }^{1}$ \\ oliveras@ugr.es \\ https://orcid.org/0000-0002-8335-698X \\ ${ }^{1}$ Universidad de Granada (UGR) \\ Granada, España.
}

Recibido: 08/04/2020 Aceptado: 20/05/2020

\section{Resumen}

En el presente artículo se muestra una investigación cuyo resultado es un singular instrumento de evaluación educativa, para aplicar a niños que comienzan la Educación Primaria, formado por 40 juegos infantiles tradicionales, tomados como herramienta evaluadora y los 50 ítems del test Boehm de Conceptos Básicos (Boehm, 1983), tomados como contenidos para la evaluación de competencias matemáticas y científicas de niños de 5 a 6 años. El estudio fue realizado en Granada (España) entre los años 2018 y 2020. Se parte de que existen capacidades matemáticas y científicas en los jugadores, que se pueden poner de manifiesto cuando se juegan, con rigor y éxito, juegos con potencialidades matemáticas y científicas. Para determinar éstas, se realizó el análisis de un conjunto de juegos tradicionales pertenecientes a diversas culturas y países del mundo, desde una perspectiva etnomatemática. Se analizaron las potencialidades matemáticas y científicas de los juegos, mediante un análisis de contenido de las reglas de juego y un análisis etnográfico de los materiales y el contexto del juego, estableciendo un catálogo de 40 juegos válidos para aprender y evaluar matemáticas y ciencias al ser jugados. Fue seleccionada una muestra de 4 juegos con potencialidades altas y fue realizado un estudio de casos, cuyos resultados permitieron validar el catálogo. Por otra parte se había analizado el test Boehm, cuando se introdujo en España, encontrando en todos sus ítems contenidos matemáticos, propios del pensamiento infantil preescolar, y obteniendo índices de dificultad para cada concepto del test (Oliveras 1984). Los resultados de ambos estudios junto al análisis de contenido de las relaciones entre los juegos con potencialidad matemática y científica y los ítems del test citado, nos han permitido crear un instrumento de evaluación de las capacidades matemáticas y científicas infantiles, que mostramos aquí.

Palabras clave: Aprendizaje a través de juegos, Etnomatemáticas, Evaluación, Educación Primaria, Test Boehm. 


\title{
Instrumento para avaliar as competências matemáticas e científicas dos alunos que iniciam o Ensino Fundamental, por meio de jogos
}

\begin{abstract}
Resumo
Este artigo mostra uma investigação cujo resultado é um instrumento único de avaliação educacional, a ser aplicado a crianças que iniciam o Ensino Fundamental, composto por 40 jogos tradicionais para crianças, utilizados como ferramenta de avaliação e pelos 50 itens do teste de Conceitos Básicos de Boehm (Boehm, 1983), tomado como conteúdo para a avaliação de habilidades matemáticas e científicas de crianças de 5 a 6 anos. O estudo foi realizado em Granada (Espanha) entre os anos de 2018 e 2020. Supõe-se que haja habilidades matemáticas e científicas nos jogadores, o que pode ser revelado quando jogos com potencial matemático e científico são jogados, com rigor e sucesso. Para determinar isso, foi realizada a análise de um conjunto de jogos tradicionais pertencentes a diferentes culturas e países do mundo, sob uma perspectiva etnomatemática. O potencial matemático e científico dos jogos foi analisado, através de uma análise de conteúdo das regras do jogo e uma análise etnográfica dos materiais e do contexto do jogo, estabelecendo um catálogo de 40 jogos válidos para aprender e avaliar matemática e ciências enquanto são jogadas. Uma amostra de 4 jogos com altas potencialidades foi selecionada e foi realizado um estudo de caso, cujos resultados validaram o catálogo. Por outro lado, o teste de Boehm foi analisado quando foi introduzido na Espanha, encontrando em todos os seus itens conteúdo matemático, típico do pensamento infantil pré-escolar, e obtendo índices de dificuldade para cada conceito do teste (Oliveras, 1984). Os resultados de ambos os estudos, juntamente com a análise de conteúdo das relações entre jogos com potencial matemático e científico e os itens do teste mencionado, permitiram criar um instrumento para avaliar as habilidades matemáticas e científicas das crianças, que mostramos aqui.
\end{abstract}

Palavras chave: Aprendizagem através de jogos, Etnomatemática, Avaliação, Ensino Fundamental, Teste de Boehm.

\section{Instrument to evaluate mathematical and scientific competences of the students who start Primary Education, through games}

\begin{abstract}
This article shows an investigation whose result is a unique educational evaluation instrument to apply to children starting Primary Education, made up of 40 traditional children's games, taken as an evaluation tool and the 50 items of the Boehm test of Basic Concepts ( Boehm, 1983), taken as content for the evaluation of mathematical and scientific skills of children from 5 to 6 years old. The study was carried out in Granada (Spain) between the years 2018 and 2020. It is assumed that there are mathematical and scientific abilities in the players, which can be demonstrated when games with mathematical and scientific potential are played, with rigor and success. To determine these, the analysis of a set of traditional games belonging to different cultures and countries of the world was carried out, from an ethnomathematical perspective. The mathematical and scientific potential of the games were analyzed, through a content analysis of the game rules and an ethnographic analysis of the materials and the context of the game, establishing a catalog of 40 valid games to learn and evaluate mathematics and science by being played. A sample of 4 games with high potentialities was selected and a case study was carried out, the results of which validated the catalog. On the other hand, the Boehm test had been analyzed when it was introduced in Spain, finding in all its items mathematical content, typical of preschool childhood thinking, and obtaining difficulty indices for each concept of the test
\end{abstract}


(Oliveras 1984). The results of both studies together with the content analysis of the relationships between games with mathematical and scientific potential and the ítems of the aforementioned test, have allowed us to create an instrument for evaluating children's mathematical and scientific abilities, which we show here.

Keywords: Learning through games, Ethnomathematics, Evaluation, Primary Education, Boehm test.

\section{Introducción}

El juego es un elemento esencial en la vida de cualquier persona. No solo nos aporta diversión, entretenimiento y una serie de valores positivos, sino que también puede ser un gran recurso de enseñanza y sobre todo un método de aprendizaje. Esta concepción toma aún más relevancia durante las primeras etapas educativas, donde el alumnado requiere de una serie de dinámicas que le faciliten el aprendizaje, la atención y también en gran medida, la motivación. Teniendo estas ideas en mente, es necesario destacar el punto de vista ofrecido por Morris, Croker, Zimmerman, Gill y Romig (2013). Estos autores ponen de manifiesto cómo, a pesar de su importancia en la sociedad actual y futura, con frecuencia, las destrezas asociadas al pensamiento científico y matemático no se desarrollan en las aulas, por lo que deben ser favorecidas a través de herramientas educativas y culturales, como son los juegos. Por ello, con esta investigación, también se pretende desterrar la creencia de que las matemáticas y las ciencias son materias aisladas del contexto social y de las metodologías lúdicas, generando en los estudiantes una actitud negativa hacia ellas.

En las próximas líneas nos centraremos en el juego como actividad cultural y, sobre todo, educativa, apoyándonos en los principios del aprendizaje lúdico y basado en juegos (Resnick, 2004; Kangas, 2010), con especial interés en su repercusión sobre la educación científica y matemática (Bergen, 2009; Chang, 2013; Vázquez-Alonso y Manassero-Mas, 2017). Tal y como afirmaba Piaget (1973), todo pensamiento surge de acciones y los conceptos matemáticos tienen su origen en los actos que el niño lleva a cabo con los objetos, de tal manera que los actos comienzan a ser interiorizados dando lugar a un conocimiento práctico. Es decir, la práctica del juego puede generar en el alumnado la creación de una serie de habilidades o competencias que desarrollen el pensamiento matemático y científico. Entendemos por competencia la pericia, aptitud o idoneidad para hacer o desarrollar actividades, es una capacidad activa para desempeñar tareas con éxito, que construye al propio sujeto humano.

Tomando estas ideas como referencia, queremos plantear que una de nuestras 
preocupaciones de investigación es destacar la importancia del juego en la educación, en este caso como factor determinante para la adquisición de competencias matemáticas y científicas, siendo a su vez el juego, un elemento que fomenta el desarrollo de las capacidades sociales y personales del sujeto, tanto como sus capacidades matemáticas y científicas.

El otro interrogante que nos asalta, es cómo se pueden ponen de manifiesto esas capacidades para tener una evaluación de cada niño al finalizar la etapa de Educación Infantil de cara al inicio de la Educación Primaria. La respuesta ha sido: a la hora de jugar; mediante la práctica de juegos podemos observar las destrezas, conceptos y competencias que poseen. Jugando y mediante otros procesos de aprendizaje, han llegado a lograr diversas capacidades y jugando podemos apreciar cuales son estas. Algo que también se plantearon Rosas, Ceric, Aparicio, Arango, Arroyo, Benavente, Escobar, Olguín, Pizarro, Ramírez, Tenorio y Véliz en 2015, preguntándose si era posible evaluar por medio de juegos dominios cognitivos en niños que atraviesan los primeros años de educación formal.

Por ello el objeto de estudio es elaborar un instrumento que permita evaluar ciertas competencias matemáticas y científicas, logradas por el alumnado al finalizar la Educación Infantil o al iniciar la Educación Primaria, mediante el uso de determinados juegos, en conjunción con otro elemento diseñado por Ann E Boehm para manifestar aprendizajes infantiles: el "Test Boehm de Conceptos Básicos” (Boehm, 1971, 1980, 2012), que fue estudiado por Oliveras (1984), demostrando su relación con conceptos y procesos matemáticos. En aquellos años se realizaron otros estudios interesantes sobre este test, como los de Narváez, (1987) y Powers, (1986).

\section{Fundamentación teórica}

Tratando de establecer un recurso apto para evaluar capacidades matemáticas iniciales es importante conocer el concepto al que nos referimos cuando hablamos de evaluación, en este caso, citando a Gil (1999) que consideraba la evaluación como una serie de reglas sociales de validación que tienen que ver con las disciplinas del conocimiento, buscamos apoyo en dos elementos clave: la importancia de las investigaciones previas sobre evaluación, y el juego, con sus reglas sociales, como método de aprendizaje. Complementa este marco de fundamentos teóricos la Etnomatemática, paradigma desde el cual enfocamos nuestra investigación.

Durante los años ochenta, el tema de la evaluación de la calidad de la Educación Infantil 
y su influencia en el desarrollo del alumnado, motivó numerosas investigaciones que planteaban instrumentos para evaluarla. En el caso de España, es destacable el estudio realizado en Sevilla (Lera-Rodríguez, 1994) donde se aplicó en todo el alumnado el test de Preescolar (De la Cruz, 1988). Este test ofrecía diferentes medidas sobre el vocabulario, conceptos matemáticos, memoria auditiva, discriminación visual, orientación espacial, y coordinación motriz. (LeraRodríguez, 2007). Por otro lado, también se ha comprobado que cuando se ha relacionado el rendimiento de los niños, con la calidad del aula, medida mediante el instrumento: Early Chilhood Environmental Rating Scale (ECERS), (Harms y Clifford, 1980), el test de conceptos matemáticos es el que muestra más y mejor que dicha calidad de la enseñanza y el rendimiento infantil se relacionan. Lo cual, nos indica que las aulas con calidad, en las cuales se realizan actividades lúdicas, dinámicas y creativas, favorecen el desarrollo de capacidades matemáticas en el alumnado. Todo ello, presenta la misma idea que plantearon Barba-Martín y López-Pastor en 2017, y De Castro Hernández en 2007, cuyos estudios pretenden ampliar una vía de trabajo que permita instaurar procesos de Evaluación Formativa y compartida en las aulas de Educación Infantil, así como instaurar propuestas educativas de calidad para Educación Infantil, basadas en la idoneidad didáctica. En este sentido, la mayoría de autores coinciden en la necesidad de realizar una evaluación a edades tempranas con el fin de mejorar la calidad educativa.

De acuerdo con esta necesidad de educación de calidad y esta forma de propiciarla, proponemos que la evaluación debe hacerse a través de diferentes fuentes de información e instrumentos de evaluación, entre los que se cuentan cuestionarios con preguntas abiertas, cuestionarios de opción múltiple, conversaciones, bitácoras o diarios y portafolios (National Council of Teachers of Mathematics NCTM, 2000; Garrison y Ehringhaus, 2008 y Gómez, 2007). Por otro lado, el National Council of Teachers of Mathematics (2000), presenta cinco estándares de procesos para favorecer la comprensión y el uso de los contenidos en diversos contextos significativos, siendo estos: la resolución de problemas, el razonamiento y la prueba, la comunicación, las conexiones y la representación. Todos ellos, elementos presentes a su vez en el juego.

Por otro lado, podemos afirmar que el juego es un elemento que favorece el aprendizaje ya que el aprendizaje se desarrolla mediante una acción y el juego lo es, pues Huizinga (1968), (citado en Toro 2013), indica que el juego es una acción y ocupación libre, que se desarrolla dentro de unos límites temporales y espaciales determinados, y Piaget (citado por Ruiz, 2005) 
propone que el aprendizaje se apoya en la acción del sujeto que aprende, o que juega. Teniendo estas ideas en mente, es importante destacar el origen de esta tendencia, que pretende innovar dentro del ámbito educativo, proponiendo el juego como metodología.

Durante finales del siglo XX y principios del XXI, la creatividad ha tenido un papel creciente en la educación (Craft, 2008). Enseñar de manera creativa significa adoptar enfoques imaginativos para hacer el aprendizaje más interesante, emocionante y efectivo (National Advisory Committee on Creative and Cultural Education, 1999). Por ello, el aprendizaje basado en juegos (Game-based Learning o GBL, en inglés) es una buena opción para estimular este tipo de enseñanza creativa. El uso de juegos en el marco escolar puede tomar como finalidad la comprensión de conceptos o la mejora de técnicas -juegos de conocimiento-, o bien la adquisición de métodos de resolución de problemas -juegos de estrategia- (Corbalán, 1997 y Deulofeu, 2001). Zhao y Linaza (2015) destacan las ventajas del uso de juegos, en su análisis del impacto positivo en la capacidad de razonamiento de escolares de educación infantil que realizan juegos. Igualmente la aportación de Evans (2009), indicando que son los rendimientos en matemáticas y ciencias los que mejoran significativamente con este tipo de aprendizaje, lo que es clave en nuestra investigación.

Haciendo referencia al ámbito científico, se puede decir que el aprendizaje basado en juegos favorece el desarrollo del pensamiento, posibilitando aprendizajes significativos (Glenberg y Robertson, 1999), también ponen de manifiesto una serie de habilidades asociadas al pensamiento lúdico, de tal forma que a través de los juegos se pueden abordar aspectos como planteamiento de objetivos y análisis de la situación actual, pasando por la divergencia o generación de ideas, hasta la convergencia en soluciones prácticas, por lo que no solo el pensamiento científico se ve "afectado" con este tipo de metodología, sino que permite además, romper las barreras y mejorar la comunicación en el grupo y generar nuevas ideas, visiones y estrategias. Es por tanto, una potente herramienta para trabajar el pensamiento innovador y desarrollar la creatividad (González-González, 2015). Además, el juego tiene una componente psicológica y muchos autores coinciden en que jugar resulta fundamental para el desarrollo de la personalidad del niño (Gallardo y Fernández, 2010; Gómez, 2012 y Montero, 2017 citados en Gallardo-López, 2018). A lo largo de la historia, el juego ha estado siempre presente en todas las culturas y sociedades, incluso en las más primitivas. Forma parte de la genética de la persona. Se nace, crece, evoluciona y vive con el juego (Paredes, 2003, p. 32). De forma que demuestra 
la evidencia de que esta metodología (Game based learning, GBL) rescata las componentes psicológica y social del juego para potenciar habilidades personales, valores culturales y sociales (Gros, 2000). Luego es necesario cierto control de las adquisiciones logradas por los niños, explicitarlas y relacionarlas con los currículos educativos, sin olvidar el poder educativo y evaluador del juego, como nos proponemos aquí.

Complementando este marco de fundamentos teóricos consideramos el enfoque desde la Etnomatemática como base imprescindible de nuestra investigación, ya que incide en el concepto de Matemáticas cuyas competencias pretendemos medir (Oliveras, 2006, 2005, 1996). Entendemos las matemáticas como un elemento triangular, con tres componentes indisociables: una ciencia formal, un producto social y cultural y un modelo personal de pensamiento (Oliveras, 2006), en esta investigación de corte Etnomatemático (FernándezOliveras, Oliveras, Albizu, 2014) tratamos de identificar ciertos componentes del modo personal de pensar de los estudiantes, tomando ciertos conceptos de la ciencia formal e introduciéndolos en el instrumento, que construimos siendo conscientes de su influencia sociocultural, en el sentido de estar constituido por juegos tradicionales de diversas culturas, con los que tratamos de observar cómo el modelo de pensamiento de cada niño incluye versiones personales de los conceptos científicos seleccionados.

Los juegos que incluimos en el instrumento han sido estudiados también desde un enfoque etnomatemático, detectando su poder de explicitación de elementos matemáticos (Espigares-Gámez et al 2019 y Fernández-Oliveras et al, 2019) con la intención de poderlos aplicar después a la elaboración de "Microproyectos lúdicos" (MPL) para desarrollar aprendizajes matemáticos y científicos en contextos educativos formales (escuelas, institutos) y no formales (ludotecas, talleres, clubs). Por ello, definimos los microproyectos como propuestas didácticas interdisciplinares que pretenden el desarrollo de competencias desde una perspectiva constructivista generando actividades basadas en signos relevantes para una o varias culturas (Fernández-Oliveras y Oliveras, 2015). En un microproyecto intervienen tanto elementos pre-activos, como son objetivos y contenidos, como otros elementos mediadores del aprendizaje, que son los contextos y recursos. De ahí su importancia, que reside en su respuesta a las necesidades, intereses y exigencias del desarrollo del niño y en que posibilita la intervención educativa. Según Delgado (2011), el método de proyectos, con carácter globalizador tiene en cuenta los conocimientos e intereses del alumnado, se basa en 
situaciones reales y es previamente planificado. Algo muy similar a lo establecido por Muñoz y Díaz (2009), quienes ponen de manifiesto la importancia de este tipo de aprendizaje como opción metodológica basada en la investigación-acción, cuyo objetivo es organizar los contenidos curriculares bajo un enfoque globalizador y significativo, relacionando los conocimientos escolares con los de la vida cotidiana.

Respondiendo así, a nuestra concepción de matemáticas etno y de educación mediante enculturación creativa, vivenciando los elementos científicos y matemáticos en contextos de la vida cotidiana y lúdicos, que hemos desarrollado en nuestras investigaciones previas (Espigares-Gámez, 2018).

\section{Metodología}

En el ámbito de las diferentes concepciones teóricas sobre el aprendizaje matemático temprano, se justifica la necesidad de contar con un instrumento de evaluación de los niveles de desarrollo matemático en alumnos y alumnas de Educación Infantil (Navarro-Guzmán et al., 2010). Por ello, tomando las ideas anteriores como referencia y base de nuestro estudio pretendemos materializarlas en forma de instrumento que permita conocer las capacidades matemáticas existentes en el alumnado que inicia Educación Primaria.

Como se ha comentado con anterioridad, para elaborar el instrumento se parte de dos elementos diferenciados, pero confluyentes y que se complementan. Por un lado tenemos nuestro propio catálogo de juegos, el cual fue obtenido como resultado de una revisión bibliográfica realizada entre los años 2018 y 2020, y un estudio de la muestra seleccionada mediante análisis de contenido, explicitando los elementos matemáticos que se incluyen en sus reglas de juegos y sus elementos materiales: fichas, tableros, espacios, tiempos. Este análisis se inició con una serie de juegos tradicionales de la cultura jamaicana recopilados en un estudio anterior (Espigares-Gámez et al 2019 y Fernández-Oliveras et al, 2019) y posteriormente fue ampliado con el resto de juegos tradicionales de diversas culturas, obtenido también a través de una revisión bibliográfica y el análisis de la segunda muestra seleccionada (Fernández-Oliveras et al, 2019). De esta forma, se logró obtener un catálogo de 40 juegos tradicionales de todo el mundo, pertenecientes a diferentes países y culturas, y tras la realización de un análisis de las potencialidades matemáticas y científicas que se prevé serán activadas al ponerlos en práctica, mostraron tener en común que ponen de manifiesto una gran variedad de competencias matemáticas y científicas que cada juego requiere para 
ser jugado con éxito. Existen muchas coincidencias en los aprendizajes posibles de potenciar durante su realización, a pesar de sus diferencias individuales.

Cabe puntualizar que muchos juegos provienen de un lugar pero se diseminan por el mundo, siendo los lugares expresados en el cuadro (Cuadro 1, dividido en C1a, C1b, C1c, C1d, C1e, por su extensión) aquellos en los que se originaron, según las fuentes consultadas, y que suelen coincidir con países en que se practican actualmente. Entre los juegos mostrados hay siete europeos, seis americanos, tres africanos, uno asiático y uno de Oceanía, por lo que están representados los cinco continentes, para promover una educación intercultural con enfoque etnomatemático (Oliveras, 2005 y Espigares-Gámez et al., 2019).

En el citado estudio de los juegos, realizamos una parte teórica consistente en generar un nuevo sistema de clasificación de juegos, (Fernández-Oliveras et al., 2019) y otra práctica consistente en su aplicación a la selección de juegos de todo el mundo elaborada mediante revisión bibliográfica, (Fernández-Oliveras et al., 2019) con lo cual obtuvimos mucha información (tipo de juego: edad, cuerpo-mente, cantidad de jugadores, entorno interiorexterior, populares-tradicionales, competitivos, cooperativos, de rol, su origen, su potencial de desarrollo científico o matemático) sobre estos, que nos permite ahora convertirlos en un instrumento evaluador de competencias científicas y matemáticas, asociadas al test Boehm.

Los datos del estudio sobre el origen geopolítico de cada juego, sus materiales y las competencias matemáticas y científicas implicadas aparecen sintetizados en el Cuadro1, se aporta web-grafía donde encontrar la descripción detallada de cada juego, (la numeración es la correspondiente al juego del Catálogo elaborado), presentado en este Cuadro 1:

Cuadro 1. C1a: Catálogo de juegos tradicionales analizados

\begin{tabular}{|l|l|l|l|}
\hline $\begin{array}{l}\text { Número y nombre del } \\
\text { Juego }\end{array}$ & $\begin{array}{l}\text { Origen o lugar } \\
\text { de uso actual }\end{array}$ & Competencias matemáticas & Competencias científicas \\
\hline $\begin{array}{l}\text { 1. ¿Qué hora es señor } \\
\text { lobo? } \\
\text { No materiales. } \\
\text { Más de } 3 \text { jugadores }\end{array}$ & Inglaterra & $\begin{array}{l}\text { Aritmética Estimación } \\
\text { Números naturales } \\
\text { Medida de distancias }\end{array}$ & $\begin{array}{l}\text { Observación Formulación de } \\
\text { hipótesis } \\
\text { Evaluación de suposiciones } \\
\text { Concepto de velocidad }\end{array}$ \\
\hline $\begin{array}{l}\mathbf{2 . 2 0 - 2 0} \\
\text { Baraja española de } 40 \\
\text { cartas y } 6 \text { fichas para cada } \\
\text { jugador. } \\
\text { Jugadores } 2 \text { o más }\end{array}$ & España & $\begin{array}{l}\text { Números naturales Orden. } \\
\text { Aritmética Cálculo mental. } \\
\text { Estimación. Estrategia }\end{array}$ & $\begin{array}{l}\text { Observación Formulación de } \\
\text { hipótesis Planificación } \\
\text { Control de los efectos de } \\
\text { variables. Toma de } \\
\text { decisiones }\end{array}$ \\
\hline $\begin{array}{l}\text { 3. Ajutatut } \\
\text { Pelota. } \\
\text { Jugadores 4 o más }\end{array}$ & América del sur & $\begin{array}{l}\text { Aritmética Cálculo mental } \\
\text { Números naturales Resolución } \\
\text { de problemas Situación } \\
\text { espacial }\end{array}$ & $\begin{array}{l}\text { Observación Planificación } \\
\text { Formulación de hipótesis } \\
\text { Evaluación de suposiciones } \\
\text { Conceptos de dinámica }\end{array}$ \\
\hline
\end{tabular}


Cuadro 1. C1b: Catálogo de juegos tradicionales analizados (Continuación)

\begin{tabular}{|c|c|c|c|}
\hline $\begin{array}{l}\text { Número y nombre del } \\
\text { Juego }\end{array}$ & $\begin{array}{l}\text { Origen o lugar } \\
\text { de uso actual }\end{array}$ & Competencias matemáticas & Competencias científicas \\
\hline $\begin{array}{l}\text { 4. Awale } \\
\text { Semillero como tablero } \\
\text { y Semillas o fichas. } \\
\text { Jugadores } 2\end{array}$ & Sur de África & $\begin{array}{l}\text { Comparación Aritmética. } \\
\text { Fracciones Cálculo mental } \\
\text { Geometría. Medida. } \\
\text { Resolución de problemas } \\
\text { Uso de la lógica }\end{array}$ & $\begin{array}{l}\text { Observación. Formulación de } \\
\text { hipótesis. Planificación } \\
\text { Control de los efectos de } \\
\text { variables. Evaluación de } \\
\text { suposiciones } \\
\text { Toma de decisiones }\end{array}$ \\
\hline $\begin{array}{l}\text { 5. Beggar my } \\
\text { neighbourgh } \\
\text { Baraja de póker. } \\
\text { Jugadores, } 2 \text { o más }\end{array}$ & Jamaica & $\begin{array}{l}\text { Números naturales Aritmética } \\
\text { Patrones Coordinabilidad }\end{array}$ & $\begin{array}{l}\text { Observación. Planificación } \\
\text { Formulación de hipótesis } \\
\text { Evaluación de suposiciones } \\
\text { Conceptos de dinámica }\end{array}$ \\
\hline $\begin{array}{l}\text { 6. Bluebird } \\
\text { No materiales. } \\
\text { Jugadores } 3 \text { o más }\end{array}$ & Jamaica & $\begin{array}{l}\text { Probabilidad. Uso de lógica } \\
\text { Resolución de problemas }\end{array}$ & Observación Planificación \\
\hline $\begin{array}{l}\text { 7. Brown Girl in the ring } \\
\text { No materiales. } \\
\text { Jugadores } 3 \text { o más }\end{array}$ & Jamaica & $\begin{array}{l}\text { Aritmética Cálculo mental } \\
\text { Números naturales } \\
\text { Resolución de problemas } \\
\text { Situación espacial }\end{array}$ & $\begin{array}{l}\text { Observación. Análisis } \\
\text { Formulación de hipótesis } \\
\text { Evaluación de suposiciones } \\
\text { Medida de distancias } \\
\text { Concepto de velocidad }\end{array}$ \\
\hline $\begin{array}{l}\text { 8. Bruck rock Stone } \\
\text { Piedra o pelota. } \\
\text { Jugadores } 3 \text { o más }\end{array}$ & Jamaica & $\begin{array}{l}\text { Patrones Resolución de } \\
\text { problemas Sentido espacial } \\
\text { Concepto de par }\end{array}$ & $\begin{array}{l}\text { Observación. Planificación } \\
\text { Formulación de hipótesis } \\
\text { Evaluación de suposiciones } \\
\text { Conceptos de dinámica }\end{array}$ \\
\hline $\begin{array}{l}\text { 9. Bull inna pen } \\
\text { No materiales. } \\
\text { Jugadores } 3 \text { o más }\end{array}$ & Jamaica & $\begin{array}{l}\text { Probabilidad Resolución de } \\
\text { problemas } \\
\text { Uso de la lógica Medida de } \\
\text { distancias }\end{array}$ & $\begin{array}{l}\text { Observación. Análisis } \\
\text { Formulación de hipótesis } \\
\text { Evaluación de suposiciones } \\
\text { Concepto de velocidad }\end{array}$ \\
\hline $\begin{array}{l}\text { 10. Buzz Fizz } \\
\text { No materiales. } \\
\text { Jugadores } 2 \text { o más }\end{array}$ & $\begin{array}{l}\text { América del } \\
\text { norte }\end{array}$ & $\begin{array}{l}\text { Números naturales. Patrones } \\
\text { Aritmética. } \\
\text { Coordinabilidad }\end{array}$ & Observación Planificación \\
\hline $\begin{array}{l}\text { 11. Cierra la caja } \\
\text { (shut the box). } \\
\text { Caja con pestañas del } 1 \text { al } \\
10 \text { y dado. } \\
\text { Jugadores } 2 \text { o más }\end{array}$ & Australia & $\begin{array}{l}\text { Estimación. Aritmética } \\
\text { Números naturales Cálculo } \\
\text { mental Probabilidad. } \\
\text { Patrones. } \\
\text { Uso de la lógica }\end{array}$ & $\begin{array}{l}\text { Observación. Análisis } \\
\text { Formulación de hipótesis } \\
\text { Evaluación de suposiciones } \\
\text { Predicción } \\
\text { Elaboración de conclusiones }\end{array}$ \\
\hline $\begin{array}{l}\text { 12. Chinesse skip } \\
\text { Goma elástica. } \\
\text { Jugadores } 3 \text { o más }\end{array}$ & Jamaica & $\begin{array}{l}\text { Números naturales. } \\
\text { Probabilidad Interpretación } \\
\text { de códigos }\end{array}$ & $\begin{array}{l}\text { Observación. Planificación. } \\
\text { Formulación de hipótesis. } \\
\text { Evaluación de suposiciones }\end{array}$ \\
\hline $\begin{array}{l}\text { 13. Damas chinas } \\
\text { Tablero de } 6 \text { puntas fichas } \\
\text { de } 6 \text { colores. } \\
\text { De } 2 \text { a } 6 \text { jugadores }\end{array}$ & Alemania & $\begin{array}{l}\text { Categorización. Creatividad } \\
\text { Estrategia. Uso de la lógica } \\
\text { Control de variables Ángulos. } \\
\text { Medida }\end{array}$ & $\begin{array}{l}\text { Observación. Predicción. } \\
\text { Análisis. Planificación } \\
\text { Evaluación de suposiciones } \\
\text { Toma de decisiones }\end{array}$ \\
\hline
\end{tabular}


Cuadro 1. C1c: Catálogo de juegos tradicionales analizados (Continuación)

\begin{tabular}{|c|c|c|c|}
\hline $\begin{array}{l}\text { Número y nombre del } \\
\text { Juego }\end{array}$ & $\begin{array}{l}\text { Origen o lugar } \\
\text { de uso actual }\end{array}$ & Competencias matemáticas & Competencias científicas \\
\hline $\begin{array}{l}\text { 14. Dandy Shandy } \\
\text { Pelota. } 6 \text { jugadores o más, } \\
\text { en dos equipos. }\end{array}$ & Jamaica & $\begin{array}{l}\text { Organización y distribución } \\
\text { espacial. Vectores Direcciones } \\
\text { en el espacio }\end{array}$ & $\begin{array}{l}\text { Observación. Análisis } \\
\text { Formulación de hipótesis } \\
\text { Predicción. Fuerza }\end{array}$ \\
\hline $\begin{array}{l}\text { 15. Daruma Otoshi } \\
\text { Jugadores } 2\end{array}$ & Japón & $\begin{array}{l}\text { Número natural. Vectores } \\
\text { Resolución de problemas }\end{array}$ & $\begin{array}{l}\text { Observación. Planificación } \\
\text { Uso de la lógica }\end{array}$ \\
\hline $\begin{array}{l}\text { 16. Dog n'bone } \\
\text { Pañuelo. } 6 \text { jugadores o } \\
\text { más, en dos equipos }\end{array}$ & Jamaica & $\begin{array}{l}\text { Categorización. Estrategia } \\
\text { Uso de lógica. Ângulos. } \\
\text { Medida de distancia }\end{array}$ & $\begin{array}{l}\text { Observación. Análisis } \\
\text { Formulación de hipótesis } \\
\text { Concepto de velocidad }\end{array}$ \\
\hline $\begin{array}{l}\text { 17. Domino } \\
\text { (Versión French) } \\
\text { Fichas de dominó. } \\
\text { Jugadores } 2 \text { o más }\end{array}$ & Jamaica & $\begin{array}{l}\text { Estimación. Números } \\
\text { naturales. Lado y rectángulo } \\
\text { Patrones. Probabilidad } \\
\text { Resolución de problemas } \\
\text { Uso de la lógica }\end{array}$ & $\begin{array}{l}\text { Observación Predicción. } \\
\text { Análisis } \\
\text { Formulación de hipótesis } \\
\text { Evaluación de suposiciones } \\
\text { Elaboración de conclusiones }\end{array}$ \\
\hline $\begin{array}{l}\text { 18. Fanorona } \\
\text { Tablero y fichas. } \\
\text { Jugadores } 2\end{array}$ & Madagascar & $\begin{array}{l}\text { Comparación. Estimación } \\
\text { Patrones. } \\
\text { Probabilidad Resolución de } \\
\text { problemas } \\
\text { Uso de la lógica } \\
\text { Uso de la creatividad }\end{array}$ & $\begin{array}{l}\text { Observación. Predicción } \\
\text { Formulación de hipótesis. } \\
\text { Planificación. Toma de } \\
\text { decisiones. Control de los } \\
\text { efectos de variables } \\
\text { Elaboración de conclusiones }\end{array}$ \\
\hline $\begin{array}{l}\text { 19. Farmers in the dell } \\
\text { No materiales. } \\
\text { Jugadores } 2 \text { o más }\end{array}$ & Jamaica & $\begin{array}{l}\text { Comparación. Estimación } \\
\text { Dirección en el espacio } \\
\text { Vectores Resolución de } \\
\text { problemas }\end{array}$ & $\begin{array}{l}\text { Observación. Planificación } \\
\text { Formulación de hipótesis } \\
\text { Evaluación de suposiciones } \\
\text { Concepto de fuerza }\end{array}$ \\
\hline $\begin{array}{l}\text { 20. Follow the arrow } \\
\text { Tablero y fichas. } \\
\text { Jugadores } 2\end{array}$ & Jamaica & $\begin{array}{l}\text { Números naturales } \\
\text { Probabilidad }\end{array}$ & Observación Planificación \\
\hline $\begin{array}{l}\text { 21. Gig spinning } \\
\text { Peonza. } \\
\text { Jugadores } 2 \text { o más }\end{array}$ & Jamaica & $\begin{array}{l}\text { Números naturales } \\
\text { Probabilidad }\end{array}$ & $\begin{array}{l}\text { Observación. Planificación } \\
\text { Formulación de hipótesis } \\
\text { Evaluación de suposiciones }\end{array}$ \\
\hline $\begin{array}{l}\text { 22. Hide an 'seek } \\
\text { No materiales. } \\
\text { Jugadores } 2 \text { o más }\end{array}$ & Jamaica & $\begin{array}{l}\text { Números naturales } \\
\text { Probabilidad } \\
\text { Medida de distancias }\end{array}$ & $\begin{array}{l}\text { Observación. Análisis } \\
\text { Formulación de hipótesis } \\
\text { Evaluación de suposiciones } \\
\text { Concepto de velocidad }\end{array}$ \\
\hline $\begin{array}{l}\text { 23. Hopscatch } \\
\text { Piedra. } \\
\text { A partir de } 1 \text { jugador }\end{array}$ & Jamaica & $\begin{array}{l}\text { Aritmética. Cálculo mental } \\
\text { Estimación. Probabilidad } \\
\text { Números naturales. Patrones } \\
\text { Resolución de problemas }\end{array}$ & $\begin{array}{l}\text { Observación. Planificación } \\
\text { Formulación de hipótesis } \\
\text { Evaluación de suposiciones } \\
\text { Interpretación de códigos }\end{array}$ \\
\hline
\end{tabular}

Continúa 
Cuadro 1. C1d: Catálogo de juegos tradicionales analizados (Continuación)

\begin{tabular}{|c|c|c|c|}
\hline $\begin{array}{l}\text { Número y nombre del } \\
\text { Juego }\end{array}$ & $\begin{array}{l}\text { Origen o lugar } \\
\text { de uso actual }\end{array}$ & Competencias matemáticas & Competencias científicas \\
\hline $\begin{array}{l}\text { 24. Hundir la flota } \\
\text { Tablero de juego y } \\
\text { fichas o papel y lápiz. } \\
\text { Jugadores } 2\end{array}$ & $\begin{array}{l}\text { América del } \\
\text { norte }\end{array}$ & $\begin{array}{l}\text { Coordenadas. Par ordenado. } \\
\text { Estrategia. Creatividad. } \\
\text { Números naturales. Lado. } \\
\text { Uso de la lógica }\end{array}$ & $\begin{array}{l}\text { Observación. Recopilación de } \\
\text { datos. Formulación de } \\
\text { hipótesis. Planificación. } \\
\text { Evaluación de suposiciones. } \\
\text { Tomar decisiones }\end{array}$ \\
\hline $\begin{array}{l}\text { 25. La Mamba } \\
\text { No materiales. } \\
\text { Jugadores } 4 \text { o más }\end{array}$ & Norte de África & $\begin{array}{l}\text { Patrones Resolución de } \\
\text { problemas Sentido espacial } \\
\text { Concepto de par }\end{array}$ & $\begin{array}{l}\text { Observación. Planificación } \\
\text { Formulación de hipótesis } \\
\text { Evaluación de suposiciones } \\
\text { Interpretación de códigos }\end{array}$ \\
\hline $\begin{array}{l}\text { 26. Loodi } \\
\text { Tablero y fichas. } \\
\text { Jugadores } 4\end{array}$ & Jamaica & $\begin{array}{l}\text { Aritmética. Cálculo mental } \\
\text { Estimación. Probabilidad } \\
\text { Números naturales. Patrones } \\
\text { Resolución de problemas } \\
\text { Uso de la lógica } \\
\text { Uso de la creatividad }\end{array}$ & $\begin{array}{l}\text { Observación. Planificación } \\
\text { Formulación de hipótesis. } \\
\text { Predicción. Control de los } \\
\text { efectos de variables. } \\
\text { Evaluación. Elaboración de } \\
\text { conclusiones Toma de } \\
\text { decisiones }\end{array}$ \\
\hline $\begin{array}{l}\text { 27. Marbles } \\
\text { Canicas. } \\
\text { Jugadores } 2 \text { o más }\end{array}$ & Jamaica & $\begin{array}{l}\text { Comparación. Estimación } \\
\text { Dirección en el espacio } \\
\text { Vectores Resolución de } \\
\text { problemas }\end{array}$ & $\begin{array}{l}\text { Observación. Análisis. } \\
\text { Predicción. Formulación de } \\
\text { hipótesis. Evaluación. } \\
\text { Elaboración de conclusiones }\end{array}$ \\
\hline $\begin{array}{l}\text { 28. Mijnlieff. } \\
\text { Tablero cuadrado y } 8 \\
\text { fichas de distinto color } \\
\text { para cada uno de los } 2 \\
\text { jugadores }\end{array}$ & $\begin{array}{l}\text { Norte de } \\
\text { Escandinavia }\end{array}$ & $\begin{array}{l}\text { Simetría. Categorización } \\
\text { Direcciones en el plano } \\
\text { Ángulos. Medidas Figuras } \\
\text { geométricas. Resolución de } \\
\text { problemas }\end{array}$ & $\begin{array}{l}\text { Observación. Análisis } \\
\text { Formulación de hipótesis } \\
\text { Planificación } \\
\text { Elaboración de conclusiones } \\
\text { Uso de la creatividad }\end{array}$ \\
\hline $\begin{array}{l}\text { 29. Molino de } 9 \\
\text { Fichas y tablero cuadrado. } \\
\text { Jugadores } 2\end{array}$ & $\begin{array}{l}\text { Imperio } \\
\text { Romano }\end{array}$ & $\begin{array}{l}\text { Cálculo mental. Estimación } \\
\text { Razonamiento. Estrategia } \\
\text { Distribución del espacio plano }\end{array}$ & $\begin{array}{l}\text { Observación. Análisis. } \\
\text { Planificación. Formulación de } \\
\text { hipótesis. Control de los } \\
\text { efectos de variables. Tomar } \\
\text { decisions. Elaboración de } \\
\text { conclusions }\end{array}$ \\
\hline $\begin{array}{l}\text { 30. Morra. } \\
\text { Manos. } \\
\text { Jugadores } 2\end{array}$ & España & $\begin{array}{l}\text { Números naturales } \\
\text { Probabilidad }\end{array}$ & $\begin{array}{l}\text { Observación Predicción. } \\
\text { Formulación de hipótesis }\end{array}$ \\
\hline $\begin{array}{l}\text { 31. Mummy Lashy } \\
\text { No materiales. } \\
\text { Jugadores } 2\end{array}$ & Jamaica & $\begin{array}{l}\text { Simetría. Observación. } \\
\text { Planificación. Resolución de } \\
\text { problemas }\end{array}$ & $\begin{array}{l}\text { Observación. Planificación. } \\
\text { Formulación de hipótesis. } \\
\text { Evaluación de suposiciones }\end{array}$ \\
\hline $\begin{array}{l}\text { 32. My mother, your } \\
\text { mother. } \\
\text { No material. } \\
\text { Jugadores } 2\end{array}$ & Jamaica & $\begin{array}{l}\text { Simetría Resolución de } \\
\text { problemas }\end{array}$ & $\begin{array}{l}\text { Observación. Planificación. } \\
\text { Formulación de hipótesis. } \\
\text { Evaluación de suposiciones }\end{array}$ \\
\hline
\end{tabular}


Cuadro 1. C1e: Catálogo de juegos tradicionales analizados (Continuación)

\begin{tabular}{|c|c|c|c|}
\hline $\begin{array}{l}\text { Número y nombre del } \\
\text { Juego }\end{array}$ & $\begin{array}{l}\text { Origen o lugar } \\
\text { de uso actual }\end{array}$ & Competencias matemáticas & Competencias científicas \\
\hline $\begin{array}{l}\text { 33. Pajaritos ( o 31) } \\
\text { Baraja española. } \\
\text { Jugadores } 2 \text { o más }\end{array}$ & Venezuela & $\begin{array}{l}\text { Números naturales. Patrones } \\
\text { Aritmética. Cálculo mental } \\
\text { Estimación. Probabilidad } \\
\text { Resolución de problemas } \\
\text { Uso de la lógica }\end{array}$ & $\begin{array}{l}\text { Observación. Recopilación de } \\
\text { datos. Análisis. Predicción } \\
\text { Formulación de hipótesis } \\
\text { Evaluación de suposiciones } \\
\text { Toma de decisiones }\end{array}$ \\
\hline $\begin{array}{l}\text { 34. Pasar por el aro } \\
\text { Aros. } \\
\text { Jugadores } 4 \text { o más }\end{array}$ & El Salvador & $\begin{array}{l}\text { Distribución del espacio. } \\
\text { Resolución de problemas } \\
\text { Uso de la lógica }\end{array}$ & $\begin{array}{l}\text { Observación. Planificación } \\
\text { Formulación de hipótesis } \\
\text { Evaluación de suposiciones }\end{array}$ \\
\hline $\begin{array}{l}\text { 35. Pass di ball } \\
\text { Pelota. } \\
\text { Jugadores } 2 \text { o más }\end{array}$ & Jamaica & $\begin{array}{l}\text { Coordenadas. Par ordenado } \\
\text { Números naturales } \\
\text { Estrategia. Uso de la lógica }\end{array}$ & $\begin{array}{l}\text { Observación. Planificación } \\
\text { Formulación de hipótesis } \\
\text { Evaluación de suposiciones }\end{array}$ \\
\hline $\begin{array}{l}\text { 36. Pass round donkey } \\
\text { (Burro) } \\
\text { No materiales. } \\
\text { Jugadores } 2 \text { o más }\end{array}$ & Jamaica & $\begin{array}{l}\text { Categorización. Estrategia } \\
\text { Uso de la lógica Control de } \\
\text { variables Ángulos. Medida }\end{array}$ & $\begin{array}{l}\text { Planificación. Evaluación. } \\
\text { Predicción. Elaboración de } \\
\text { conclusiones. } \\
\text { Uso de la creatividad. } \\
\text { Toma de decisiones }\end{array}$ \\
\hline $\begin{array}{l}\text { 37. Rayuela } \\
\text { Piedra y suelo. } \\
\text { A partir de } 1 \text { jugador }\end{array}$ & Sur de Europa & $\begin{array}{l}\text { Estimación de distancias } \\
\text { Números naturales } \\
\text { Distribución del espacio }\end{array}$ & $\begin{array}{l}\text { Observación. Planificación } \\
\text { Formulación de hipótesis } \\
\text { Evaluación de suposiciones }\end{array}$ \\
\hline $\begin{array}{l}\text { 38. River bank game } \\
\text { No materiales. } \\
\text { Jugadores } 4 \text { o más }\end{array}$ & Jamaica & $\begin{array}{l}\text { Direcciones en el plano } \\
\text { Ángulos. Medidas Formas } \\
\text { geométricas. Lado } \\
\text { Interpretación de códigos }\end{array}$ & $\begin{array}{l}\text { Observación Formulación de } \\
\text { hipótesis. Planificación } \\
\text { Evaluación de suposiciones }\end{array}$ \\
\hline $\begin{array}{l}\text { 39. Saca yunya } \\
\text { Piedras o semillas. } \\
\text { Jugadores } 3 \text { o más }\end{array}$ & Perú & $\begin{array}{l}\text { Comparación. Estimación } \\
\text { Dirección en el espacio } \\
\text { Vectores Resolución de } \\
\text { problemas }\end{array}$ & $\begin{array}{l}\text { Observación. Formulación de } \\
\text { hipótesis. Planificación. } \\
\text { Control. Evaluación de } \\
\text { suposiciones Concepto de } \\
\text { fuerza }\end{array}$ \\
\hline $\begin{array}{l}\text { 40.S-T-O-P } \\
\text { Pelota. } \\
\text { Jugadores } 3 \text { o más }\end{array}$ & Jamaica & $\begin{array}{l}\text { Coordenadas. Números } \\
\text { naturales. Estrategia } \\
\text { Uso de la lógica } \\
\text { Uso de la creatividad }\end{array}$ & $\begin{array}{l}\text { Observación. Planificación. } \\
\text { Predicción. Formulación de } \\
\text { hipótesis. Elaboración de } \\
\text { conclusiones. } \\
\text { Toma de decisions }\end{array}$ \\
\hline
\end{tabular}

Variables que se exponen: origen o lugar de uso actual y competencias matemáticas y científicas que cada juego requiere para ser jugado con éxito. (Fuente: Elaboración propia de las autoras).

Se toman los juegos presentados sucintamente en este Cuadro 1 para elaborar el instrumento de evaluación, se pueden consultar más detalles de la dinámica de dichos juegos en las páginas webs incluidas en la webgrafía del final de este documento.

Por otro lado, se toma como referencia el test Boehm. Este instrumento creado por Ann 
E Boehm en 1967, fue elaborado para apreciar el dominio que los niños poseen de cierto número de conceptos que parecen fundamentales para la escuela durante los primeros años (Boehm, 2000). Los conceptos seleccionados se desarrollan desde muy temprana edad y resultan esenciales para la adquisición posterior de conceptos más complejos (Boehm, 2012). El test, consta de 50 ítems, uno para cada concepto, tiene un formato gráfico constituido por dibujos trazados sobre papel blanco representando situaciones en las que se involucra un concepto, las representaciones de cada ítem se presentan repartidas en dos cuadernillos, cada uno de 25 ítems, que se implementan mediante preguntas orales relacionadas con cada dibujo, realizadas en grupo a los niños por un adulto, un ejemplo de ítems y sus preguntas se muestra en las figuras $1,2,3,4$.

Figura 1: Representación de uno de los ítems más fáciles. Ítem 41 "Por encima"

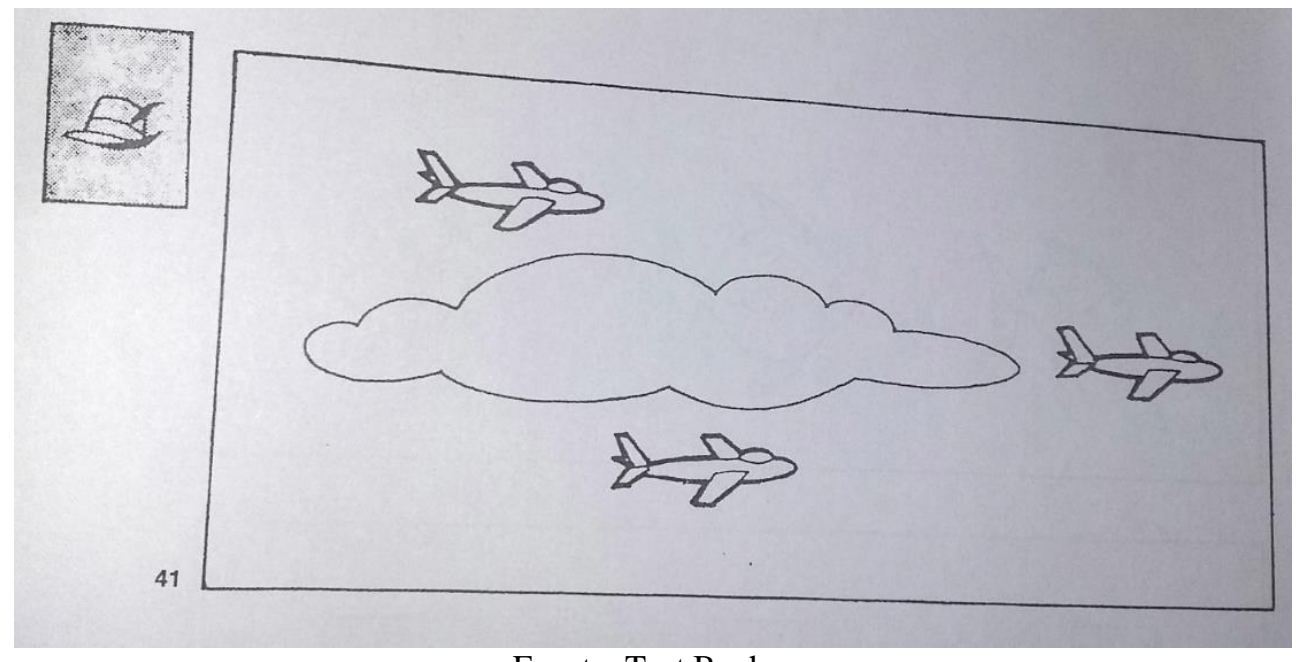

Fuente: Test Boehm

Figura 2: Instrucción oral de uno de los ítems más fáciles. Ítem 41 "Porencima".

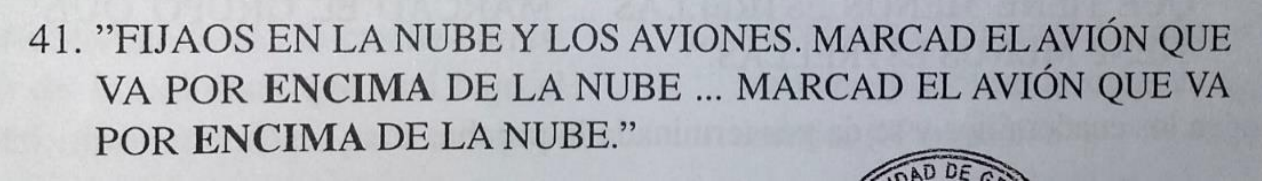

Fuente: Test Boehm 
Figura 3: Representación de uno de los ítems más complejos. Ítem 45 "Par".

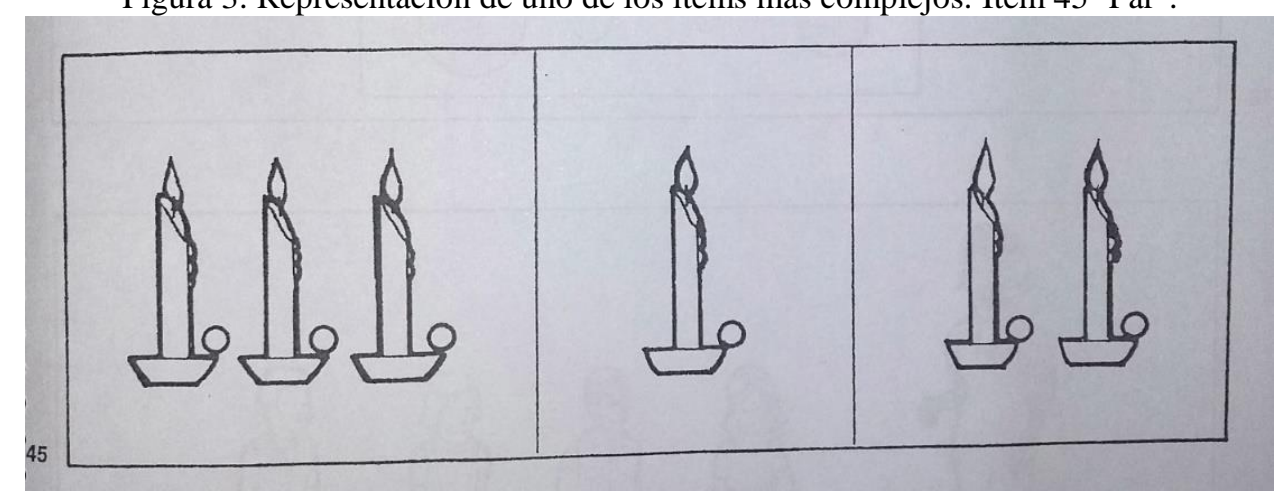

Fuente: Test Boehm

Figura 4: Instrucción oral de uno de los ítems más complejos. Ítem 45 "Par".

\section{5. "FIJAOS EN LOS CUADROS DONDE HAY VELAS DIBUJADAS. MARCAD EL CUADRO DONDE HAY UN PAR DE VELAS ... MAR- CAD EL CUADRO DONDE HAY UN PAR DE VELAS."}

\section{Fuente: Test Boehm}

Este instrumento, diseñado en Estados Unidos y validado en España en los años ochenta, que mediante un estudio realizado por Oliveras (1984) fue considerado válido para detectar capacidades matemáticas establecidas en el currículo escolar de Educación Infantil español, pensamos que tiene el valor de haber seleccionado rigurosamente 50 contenidos matemáticos básicos de carácter universal o común a todas las culturas.

Este test, también aclara cómo establecer y llevar a cabo programas de evaluación del desarrollo, de base amplia (Brassard y Boehm, 2007). Es decir, el test de conceptos básicos evalúa los conceptos fundamentales para poder seguir las instrucciones del profesor, así como para desarrollar actividades lingüísticas y matemáticas, entre otras actualmente denominadas STEAM (Boehm, 2012).

En el citado estudio de la relación de los ítems del test Boehm con elementos de las matemáticas escolares, en el punto 5.2, se mostró que "todos los ítems del test se refieren a conceptos básicos matemáticos" (Oliveras, 1984, p.118). También se observó que los tres bloques temáticos de matemáticas del currículo de Educación Infantil de entonces, denominados 'Programas Renovados', consistentes en: Lógica, relaciones, orden y equivalencia; Espacio, regiones, situaciones y referencias; Números, cantidades y medida, estaban representados en el test, aunque con diferente extensión, respectivamente 12, 21 y 17 ítems, es decir el 24\%, el 42\% y el 34\% del total de los ítems. La tabla 1 recoge los datos de Oliveras 1984 (pp.119, 120): 


\section{Tabla 1}

Índices de dificultad I.D. de cada ítem del Test Boehm, definidos en \% de aciertos.

DATOS OBTENIDOS AL APLICAR EL TEST BOEHM EN GRANADA, 1983.

(INFANTIL, N=273) (PRIMERO, N=623)

\begin{tabular}{|c|c|c|c|}
\hline ITEM & CONCEPTO & INFANTIL N=273_I.D. \% & PRIMERO E.G.B. $\mathrm{N}=623$ _I.D. $\%$ \\
\hline 1 & Arriba & 86,81 & 91,81 \\
\hline 2 & A través & 82,05 & 89,24 \\
\hline 3 & Lejos & 83,88 & 89,72 \\
\hline 4 & Junto a & 91,57 & 95,82 \\
\hline 5 & Dentro & 94,87 & 95,98 \\
\hline 6 & Algunas, pero pocas & 92,30 & 95,34 \\
\hline 7 & Medio & 92,67 & 97,75 \\
\hline 8 & Pocas & 78,02 & 82,50 \\
\hline 9 & Más lejos & 93,40 & 98,07 \\
\hline 10 & Alrededor & 93,97 & 97,11 \\
\hline 11 & Encima & 95,98 & 97,91 \\
\hline 12 & Más ancha & 46,88 & 52,00 \\
\hline 13 & Más & 83,51 & 92,13 \\
\hline 14 & Entre & 84,61 & 78,65 \\
\hline 15 & Entera & 98,53 & 97,75 \\
\hline 16 & Más cerca & 91,94 & 97,59 \\
\hline 17 & Segundo & 82,78 & 94,54 \\
\hline 18 & Esquina & 79,12 & 87,31 \\
\hline 19 & Varios & 86,08 & 95,96 \\
\hline 20 & Detrás & 90,10 & 95,18 \\
\hline 21 & Fila & 84,24 & 86,31 \\
\hline 22 & Diferente & 63,36 & 78,65 \\
\hline 23 & Después & 67,76 & 61,79 \\
\hline 24 & Casi & 71,79 & 80,09 \\
\hline 25 & Mitad & 75,82 & 86,03 \\
\hline 26 & Centro & 80,95 & 90,36 \\
\hline 27 & Tantas & 80,21 & 87,80 \\
\hline 28 & Lado & 73,62 & 70,30 \\
\hline 29 & Empezado & 57,87 & 65,97 \\
\hline 30 & Otro & 80,58 & 89,88 \\
\hline 31 & Semejante & 62,27 & 65,00 \\
\hline 32 & Ni primero ni último & 88,64 & 90,85 \\
\hline 33 & Nunca & 85,34 & 85,23 \\
\hline 34 & Debajo & 95,23 & 96,95 \\
\hline 35 & Hace pareja & 65,56 & 76,24 \\
\hline 36 & Siempre & 79,85 & 82,98 \\
\hline 37 & Tamaño mediano & 87,17 & 90,69 \\
\hline 38 & Derecha & 61,90 & 74,15 \\
\hline 39 & Delante & 69,23 & 78,00 \\
\hline 40 & Cero & 78,75 & 91,33 \\
\hline 41 & Por encima & 91,47 & 98,07 \\
\hline 42 & Cada & 73,99 & 70,46 \\
\hline 43 & Separadas & 83,51 & 87,64 \\
\hline 44 & Izquierda & 62,63 & 71,74 \\
\hline 45 & Par & 43,58 & 25,68 \\
\hline 46 & Saltarse & 50,91 & 75,28 \\
\hline 47 & Igual & 63,03 & 75,28 \\
\hline 48 & En orden & 52,38 & 69,18 \\
\hline 49 & Tercero & 45,05 & 43,33 \\
\hline 50 & Menos & 74,35 & 90,20 \\
\hline
\end{tabular}

Se consideran Idóneos para la edad y nivel escolar los ítems cuyo I.D. es superior al 70\%, y difíciles o muy dificiles aquellos de I.D. inferior, que aparecen coloreados en la tabla. (Fuente: Oliveras, 1984) 
Se estudió el índice de dificultad de cada ítem, en términos de \% de aciertos en las dos muestras de alumnado de Preescolar y $1^{\circ}$ de Primaria, obteniéndose los resultados expuestos.

Se han coloreado, en dicha Tabla 1 , los ítems que resultaron no idóneos, o sea difíciles (50\%-70\% de aciertos) o muy difíciles (I.D. inferiores al 50\%), en cada muestra o nivel escolar, que por su menor tanto por ciento de aciertos requieren una especial atención en las actividades de aprendizaje, o bien en el momento de su evaluación, siendo conveniente comprobar su logro mediante varios elementos de control, ya que pudiera depender su éxito del instrumento evaluador. Los restantes ítems muestran porcentajes elevados de acierto y son conceptualizados como conceptos básicos que resultan fáciles (80\%-90\%) o muy fáciles (90\% de aciertos en adelante).

Se puede observar que algunos ítems tienen un I.D. más bajo en primero de Primaria que en Educación Infantil (Tabla 2), ocurriendo esto en cinco ítems idóneos y en tres difíciles, lo que indica que esta diferencia de porcentaje de aciertos no es imputable a la dificultad de los conceptos medidos. Las diferencias son poco significativas sin embargo ponen de manifiesto una realidad captada por el profesorado: el efecto vacaciones produce en los niños olvidos o disminución de la atención escolar, ya que las pruebas del test se efectuaron al final del curso en Infantil y al comienzo del curso en Primaria, mediando las vacaciones de verano, las más largas en el calendario escolar español. También puede ocurrir que algunos niños de primer curso de Primaria no hayan tenido escolarización en Infantil, ya que no era obligatoria en esa fecha.

\section{Tabla 2}

Ítems que tienen un I.D más bajo en Primero de Educación Primaria que en Educación Infantil

\begin{tabular}{cccc}
\hline ÍTEM & CONCEPTO & $\begin{array}{c}\text { INFANTIL N=273 } \\
\text { I.D. \% }\end{array}$ & $\begin{array}{c}\text { PRIMERO E.G.B. } \\
\text { N=623_I.D.\% }\end{array}$ \\
\hline 14 & Entre & 84,61 & 78,65 \\
15 & Entera & 98,53 & 97,75 \\
23 & Después & 67,76 & 61,79 \\
28 & Lado & 73,62 & 70,30 \\
33 & Nunca & 85,34 & 85,23 \\
42 & Cada & 73,99 & 70,46 \\
45 & Par & 43,58 & 25,68 \\
49 & Tercero & 45,05 & 43,33 \\
\hline
\end{tabular}

Se consideran Difíciles los ítems cuyo I.D. es inferior al 70\%, que aparecen coloreados en la tabla 2 , siendo más difíciles en primero de Primaria que al finalizar Infantil. (Fuente: Oliveras1984) 
El test Boehm presenta a nuestro entender un fallo grave consistente en pretender evaluar mediante representaciones planas realidades que son tridimensionales, en una edad en que los niños no tienen construido el espacio proyectivo, lo que condiciona las interpretaciones de dibujos que tratan de representar el mundo tridimensional en que vivimos.

En Oliveras 1984, (pp.116) también se indica que hay que realizar una revisión completa del test ya que presenta serias dificultades en cuanto a la forma de implementarlo pues requiere explicaciones orales acordes con las fichas que presentan una situación representada de forma gráfica y estos dos elementos ya no son tan universales ni independientes del énfasis del encuestador. Por estos motivos hemos ido concibiendo la idea de elaborar una alternativa a los aspectos oral y gráfico del test, manteniendo los conceptos a evaluar, y hemos llegado a concluir que el mejor recurso para esa alternativa son los juegos.

Combinando estos dos elementos: Test Boehm y Catálogo de juegos, mediante un análisis de contenido que permita el establecimiento de relaciones recíprocas, se pretende conocer qué ítems del test poseen los juegos seleccionados, como 'potencial de desarrollo matemático', incluido en lo que llamamos potencial de desarrollo STEAM (PDS) y que está concebido dentro del pensamiento matemático-científico (Fernández-Oliveras, EspigaresGámez y Oliveras, 2020). Con el fin de afirmar que se dominan los conceptos matemáticos del test que han resultado asociados a su PDS (Fernández-Oliveras y Oliveras, 2014, 2015; Espigares-Gámez, Fernández-Oliveras y Oliveras, 2019).

Realizamos ambos análisis de contenido, de los juegos en las investigaciones previas citadas y del test en esta, mediante estudio de los aspectos matemáticos de las interrogantes orales y de las representaciones pictóricas, de modo que puedan ser puestas en relación con un solo concepto y establecimos las relaciones pertinentes entre ambas componentes, test y catálogo de juegos, obteniendo unas relaciones múltiples muy prometedoras de cara a la evaluación de las capacidades infantiles, la representación de estas relaciones se presenta en el cuadro 2, como resultados, a continuación.

En último lugar hay que hacer constar que somos conscientes de que en el estudio de los juegos establecimos habilidades, destrezas o competencias matemáticas y científicas, y no conceptos o contenidos, que estuvieran implícitos en la práctica de los juegos. Mientras que el test trata de conceptos. Sin embargo no existe disidencia al relacionarlos, ya que consideramos que el dominio de un concepto por un niño no se explicita mediante declaraciones definitorias 
del mismo, sino a través de destrezas, habilidades o competencias, que son observables, y que involucren a dicho concepto.

En este sentido consideramos que hay una relación inclusiva de los conceptos en las habilidades y destrezas y de estas en las competencias, que es conveniente establecer sucintamente, indicando lo que significan para nosotros. Creemos que las competencias son un conjunto de destrezas, habilidades, conocimientos y actitudes propias de una persona, que se movilizan conjuntamente para lograr una acción eficaz.

Este concepto que surgió en el ámbito laboral, pasó al didáctico, donde una de las concepciones es la del enfoque socioeducativo, en el que se plantea una competencia como una actuación integral para identificar, interpretar, argumentar y resolver problemas del contexto con idoneidad y compromiso ético, articulando el saber ser, el saber hacer y el saber conocer (García Fraile y Tobón, 2009).

También las competencias son entendidas como un saber hacer en situaciones concretas que requieren la aplicación creativa, de conocimientos, habilidades y destrezas (Delors, 1996). Para Perrenoud: 'El concepto de competencia se refiere a la manera que permite hacer frente, regular y adecuadamente, a un conjunto de tareas y de situaciones, haciendo apelación a las nociones, los conocimientos, a las informaciones, a los procedimientos, los métodos, las técnicas y también a las otras competencias más específicas’'(Perrenoud, 2008, p. 3).

En nuestro caso las competencias son específicamente matemáticas y científicas por lo que incluyen conceptos, procedimientos y actitudes propias de las ciencias experimentales y de las matemáticas.

\section{Resultados y discusión}

Aclarada la inclusión de los conceptos en las competencias, aludimos a que hemos establecido el conjunto de conceptos y competencias matemáticas y científicas que reúne el conjunto de juegos (presentados en el Cuadro1), según los estudios previos realizados sobre ellos.

En el presente estudio hemos clasificado los conceptos y competencias matemáticas y científicas en bloques correspondientes a distintos campos de las matemáticas, y asignado a cada elemento matemático o científico los ítems del test que corresponden a ese campo. Es decir en el siguiente cuadro (Cuadro 2), mostramos el resultado del análisis de contenido de las relaciones entre los ítems del test y las competencias de los juegos, lo que nos permite establecer qué juegos 
pueden evaluar cada ítem, por tener afinidad de campo conceptual, siendo esta relación el fundamento del instrumento evaluador.

El instrumento evaluador está constituido por:

1. El Cuadro 3, dividido en Cuadro 3a y Cuadro3b, (su extensión obliga a dividirlo) que muestra la relación de los ítems con los juegos del catálogo elaborado;

2. El Protocolo de observación, la Ficha de reflexión y la Rúbrica de valoración, que son los recursos diseñados para hacer operativa la aplicación del cuadro 3.

Cuadro 2: Competencias matemáticas y científicas del conjunto de juegos agrupadas en Campos, e ítems del test Boehm que corresponden a cada Campo

\begin{tabular}{|l|l|l|}
\hline Competencias matemáticas y científicas /Ítems & \multicolumn{2}{|l|}{ Ítems del Test Boehm } \\
\hline Campo Numérico: & 1. Arriba & 27. Tantas \\
Aritmética: $6,8,19$ & 2. A través & 28. Lado \\
Coordinabilidad: $27, \mathbf{4 2}$ & 3. Lejos & 29. Empezando \\
Estimación: 13,24 & 4. Junto a & 30. Otro \\
Números naturales: 40 & 5. Dentro & 31. Semejante \\
Medida: 37,50 & 6.Alguna, & 32. Ni primero, \\
Medida de distancias: 12,16 & pero pocas & ni último \\
Fracciones: $7,15,25$ & 7. Medio & 33. Nunca \\
Concepto de par: $35, \mathbf{4 2}, 45$ & 8. Pocas & 34. Debajo \\
& 9. Más Lejos & 35. Hace pareja \\
Campo Lógico: Uso de la lógica & 10. Alrededor & 36. Siempre \\
Comparación: 22 & 11. Encima & 37. Tamaño mediano \\
Patrones: 48 & 12. Más ancha & 38. Derecha \\
Interpretación de códigos: $\mathbf{3 3}$ & 13. Más & 39. Adelante \\
Orden: $17,21,23,29,32, \mathbf{4 6}, 49$ & 14. Entre & 40. Cero \\
Categorización: $30,31, \mathbf{3 3}, 36,47$ & 15. Entera & $\mathbf{4 1 . ~ P o r ~ e n c i m a ~}$ \\
& 16. Más cerca & $\mathbf{4 2 . ~ C a d a ~}$ \\
Campo Espacial y Geométrico: & 17. Segundo. & 43. Separadas \\
Sentido espacial: $1, \mathbf{2}$ & 18. Esquina & 44. Izquierda \\
Dirección en el espacio. Vector: $38, \mathbf{3 9}, 44$ & 19. Varios & 45. Par \\
Situación espacial: $3,4,5,9,11,20,34, \mathbf{4 1}$ & 20. Detrás & 46. Saltarse \\
Distribución del espacio: $10,14,43$ & 21. Fila & 47. Igual \\
Figuras geométricas, elementos: $18,26,28$ & 22. Diferente & 48. En orden \\
Campo Científico-experimental & 23. Después & 49. Tercero \\
Conceptos de dinámica: 39, 41, 46 & 24. Casi & 50. Menos \\
Concepto de fuerza (y dirección): $\mathbf{2}$ & 25. Mitad & \\
Observación: todos los ítems & 26. Centro & \\
Análisis: todos los ítems & & \\
Toma de decisiones: todos los ítems & & \\
\hline
\end{tabular}

Conceptos y competencias matemáticas y científicas clasificados en los siguientes campos: Numérico, Lógico, Espacial y Geométrico, Científico-experimental. Hay varios ítems que corresponden a dos campos a la vez, lo que hemos significado con su número en negrita. Fuente: Elaboración propia de las autoras

Aunque en los juegos (según el estudio de ellos) hay más competencias agrupadas en campos matemáticos y científicos que no se asocian con ítems del test, solo hemos representado en el cuadro 2 los campos que tienen asociado algún ítem de dicho test.

Queremos resaltar que todos los ítems del test requieren, para ser contestados 
adecuadamente, elementos que corresponden al campo científico, como son: la observación, el análisis y la toma de decisiones; también que hay varios ítems que corresponden a dos campos a la vez, lo que hemos significado poniendo su número en negrita en el cuadro, y especialmente importa resaltar que todos los ítems encuentran su significado en alguno de los conceptos o competencias de los campos extraídos de los juegos.

Se observan pocos ítems correspondientes a conceptos científicos, mientras que hay tres elementos metodológicos de la ciencia (Observación, Análisis, Toma de decisiones), relacionados con todos los ítems. Sin embargo lo importante es que todos los ítems guardan relación con al menos uno de los campos científico o matemáticos emanados de los juegos, como ya hemos indicado, lo que nos garantiza que puede ser evaluada la adquisición del concepto del ítem mediante la práctica de alguno de dichos juegos.

Tenemos que mostrar cuales son los juegos adecuados para realizar la evaluación de cada uno de los ítems, lo que hacemos a continuación, mediante el Cuadro 3, que hemos dividido en dos: Cuadro 3 a que representa a los 20 primeros juegos y Cuadro $3 b$ que representa a los juegos numerados desde el 21 al 40 (del Cuadro 1).

La evaluación del cumplimiento de los ítems, se realizará mediante la práctica organizada de cada juego con la guía de un monitor o profesor y con elementos de recogida de información, cómo el Protocolo de Observación del éxito en el desarrollo del juego (Cuadro 5), y la Ficha de Reflexión y representación de la vivencia experimentada al jugar (Cuadro 6), que serán valorados mediante una Rúbrica, diseñada para ello (Cuadro 7), todo elaborado como parte de esta investigación y que mostraremos a continuación.

En el siguiente Cuadro 3 (dividido en dos por su extensión: Cuadro 3a y Cuadro 3b) aparecen reflejados el Catálogo con los 40 juegos tradicionales, situados en el eje de abscisas y el test Boehm, con sus 50 ítems en el eje de ordenadas. En la intersección entre estos aparecen reflejadas mediante el icono " $\mathrm{X}$ ", las relaciones entre los ítems y los juegos, es decir los conceptos del test que se ponen de manifiesto en cada juego popular. En la parte inferior del cuadro, en la última fila, aparece el recuento de los ítems que se han manifestado en cada juego. A su vez en la última columna, a la derecha, se muestra el recuento de cuantos juegos del catálogo permiten evaluar cada ítem del test Boehm. Los siguientes Cuadros C3a y C3b, se sintetizarán en el Cuadro 4 para realizar un análisis numérico de sus datos. En las figuras: 5, 6, 7, se muestran fotos de ejemplos de juegos del Catálogo elaborado (Cuadro 1). 
Cuadro 3. C 3a: Relaciones entre los juegos tradicionales del catálogo elaborado y los conceptos del test Boehm

\begin{tabular}{|c|c|c|c|c|c|c|c|c|c|c|c|c|c|c|c|c|c|c|c|c|c|}
\hline Ítems & 1 & 2 & 3 & 4 & 5 & 6 & 7 & 8 & 9 & 10 & 11 & 12 & 13 & 14 & 15 & 16 & 17 & 18 & 19 & 20 & Total \\
\hline 1. Arriba & & & & & & & & $\mathrm{X}$ & & & & & & & & & & & & & 1 \\
\hline 2. A través & & & & & & & & $\mathrm{X}$ & & & & & & & & & & & & & 1 \\
\hline 3. Lejos & & & $\mathrm{X}$ & & & & $\mathrm{X}$ & & & & & & & $\mathrm{X}$ & & & & & & & 3 \\
\hline 4. Junto a & & & $\mathrm{X}$ & & & & $\mathrm{X}$ & & & & & & & $\mathrm{X}$ & & & & & & & 3 \\
\hline 5. Dentro & & & $\mathrm{X}$ & & & & $\mathrm{X}$ & & & & & & & $\mathrm{X}$ & & & & & & & 3 \\
\hline $\begin{array}{l}\text { 6. Algunas, } \\
\text { pero pocas }\end{array}$ & $\mathrm{X}$ & & $\mathrm{X}$ & $\mathrm{X}$ & $\mathrm{X}$ & & $\mathrm{X}$ & & & $\mathrm{X}$ & $\mathrm{X}$ & & & & & & & & & & 7 \\
\hline 7. Medio & & & & $\mathrm{X}$ & & & & & & & & & & & & & & & & & 1 \\
\hline 8. Pocas & $\mathrm{X}$ & & $\mathrm{X}$ & $\mathrm{X}$ & $\mathrm{X}$ & & $\mathrm{X}$ & & & $\mathrm{X}$ & $\mathrm{X}$ & & & & & & & & & & 7 \\
\hline 9. Más Lejos & & & $\mathrm{X}$ & & & & $\mathrm{X}$ & & & & & & & $\mathrm{X}$ & & & & & & & 3 \\
\hline 10. Alrededor & & & & & & & & & & & & & & $\mathrm{X}$ & & & & & & & 1 \\
\hline 11. Encima & & & $\mathrm{X}$ & & & & $\mathrm{X}$ & & & & & & & $\mathrm{X}$ & & & & & & & 3 \\
\hline $\begin{array}{l}\text { 12. Más } \\
\text { ancha }\end{array}$ & $X$ & & & & & & & & $\mathrm{X}$ & & & & & & & $X$ & & & & & 3 \\
\hline 13. Más & $X$ & $\mathrm{X}$ & & & & & & & & & $X$ & & & & & & $X$ & $X$ & $X$ & & 6 \\
\hline 14. Entre & & & & & & & & & & & & & & $\mathrm{X}$ & & & & & & & 1 \\
\hline 15. Entera & & & & $\mathrm{X}$ & & & & & & & & & & & & & & & & & 1 \\
\hline 16. Más cerca & $\mathrm{X}$ & & & & & & & & $\mathrm{X}$ & & & & & & & $X$ & & & & & 3 \\
\hline 17 Segundo. & & $\mathrm{X}$ & & & & & & & & & & & & & & & & & & & 1 \\
\hline 18. Esquina & & & & & & & & & & & & & $\mathrm{X}$ & & & & $\mathrm{X}$ & & & & 2 \\
\hline 19. Varios & $\mathrm{X}$ & & $\mathrm{X}$ & $\mathrm{X}$ & $\mathrm{X}$ & & $\mathrm{X}$ & & & $\mathrm{X}$ & $\mathrm{X}$ & & & & & & & & & & 7 \\
\hline 20. Detrás & & & $\mathrm{X}$ & & & & $\mathrm{X}$ & & & & & & & $\mathrm{X}$ & & & & & & & 3 \\
\hline 21. Fila & & $\mathrm{X}$ & & & & & & & & & & & & & & & & & & & 1 \\
\hline 22. Diferente & & & & & & & & & & & & & & & & & & $\mathrm{X}$ & $\mathrm{X}$ & & 2 \\
\hline 23. Después & & $\mathrm{X}$ & & & & & & & & & & & & & & & & & & & 1 \\
\hline 24. Casi & $\mathrm{X}$ & $\mathrm{X}$ & & & & & & & & & $\mathrm{X}$ & & & & & & $\mathrm{X}$ & $\mathrm{X}$ & $\mathrm{X}$ & & 6 \\
\hline 25. Mitad & & & & $\mathrm{X}$ & & & & & & & & & & & & & & & & & 1 \\
\hline 26. Centro & & & & & & & & & & & & & $\mathrm{X}$ & & & & $\mathrm{X}$ & & & & 2 \\
\hline 27. Tantas & & & & & $\mathrm{X}$ & & & & & $\mathrm{X}$ & & & & & & & & & & & 2 \\
\hline 28. Lado & & & & & & & & & & & & & $\mathrm{X}$ & & & & $\mathrm{X}$ & & & & 2 \\
\hline 29.Empezado & & $\mathrm{X}$ & & & & & & & & & & & & & & & & & & & 1 \\
\hline 30. Otro & & & & & & & & & & & & & $\mathrm{X}$ & & & $\mathrm{X}$ & & & & & 2 \\
\hline 31. Semejante & & & & & & & & & & & & & $\mathrm{X}$ & & & $X$ & & & & & 2 \\
\hline $\begin{array}{l}\text { 32.Niprimero, } \\
\text { ni último }\end{array}$ & & $\mathrm{X}$ & & & & & & & & & & & & & & & & & & & 1 \\
\hline 33. Nunca & & & & & & & & & & & & $X$ & $\mathrm{X}$ & & & $\mathrm{X}$ & & & & & 3 \\
\hline 34. Debajo & & & $\mathrm{X}$ & & & & $\mathrm{X}$ & & & & & & & $\mathrm{X}$ & & & & & & & 3 \\
\hline $\begin{array}{l}35 . \text { Hace } \\
\text { pareja }\end{array}$ & & & & & & & & $\mathrm{X}$ & & & & & & & & & & & & & $\mathbf{1}$ \\
\hline 36. Siempre & & & & & & & & & & & & & $\mathrm{X}$ & & & $X$ & & & & & 2 \\
\hline $\begin{array}{l}\text { 37.Tamaño } \\
\text { mediano }\end{array}$ & & & & $\mathrm{X}$ & & & & & & & & & $\mathrm{X}$ & & & & & & & & 2 \\
\hline 38. Derecha & & & & & & & & & & & & & & $\mathrm{X}$ & & & & & & & 1 \\
\hline 39. Adelante & & & $X$ & & $X$ & & & $X$ & & & & & & $X$ & & & & & & & 4 \\
\hline 40. Cero & $X$ & $\mathrm{X}$ & $X$ & & $\mathrm{X}$ & & $\mathrm{X}$ & & & $\mathrm{X}$ & $\mathrm{X}$ & $\mathrm{X}$ & & & $\mathrm{X}$ & & $\mathrm{X}$ & & & $\mathrm{X}$ & 11 \\
\hline $\begin{array}{l}\text { 41. Por } \\
\text { encima } \\
\end{array}$ & & & $\mathrm{X}$ & & $\bar{X}$ & & $\mathrm{X}$ & $\mathrm{X}$ & & & & & & $\mathrm{X}$ & & & & & & & 5 \\
\hline 42. Cada & & & & & $\mathrm{X}$ & & & $\mathrm{X}$ & & $\mathrm{X}$ & & & & & & & & & & & 3 \\
\hline 43. Separadas & & & & & & & & & & & & & & $\mathrm{X}$ & & & & & & & 1 \\
\hline 44. Izquierda & & & & & & & & & & & & & & $\mathrm{X}$ & & & & & & & 1 \\
\hline 45. Par & & & & & & & & $\mathrm{X}$ & & & & & & & & & & & & & 1 \\
\hline 46. Saltarse & & $\mathrm{X}$ & $\mathrm{X}$ & & $\mathrm{X}$ & & & $\mathrm{X}$ & & & & & & & & & & & & & 4 \\
\hline 47. Igual & & & & & & & & & & & & & $\mathrm{X}$ & & & $\mathrm{X}$ & & & & & 2 \\
\hline 48. En orden & & & & & & & & $\mathrm{X}$ & & $\mathrm{X}$ & $\mathrm{X}$ & & & & & & $\mathrm{X}$ & $\mathrm{X}$ & & & 5 \\
\hline 49. Tercero & & $\mathrm{X}$ & & & & & & & & & & & & & & & & & & & 1 \\
\hline 50. Menos & & & & $\mathrm{X}$ & & & & & & & & & $\mathrm{X}$ & & & & & & & & 2 \\
\hline Total ítem/J & 8 & 10 & 14 & 8 & 9 & $\overline{\mathbf{0}}$ & 12 & 9 & 2 & 7 & 7 & 2 & 10 & 14 & 1 & 7 & 7 & 4 & 3 & 1 & \\
\hline
\end{tabular}

Resultados del análisis de contenido. Fuente: Elaboración propia de las autoras 
Cuadro 3. C 3b: Relaciones entre los juegos tradicionales del catálogo elaborado y los conceptos del test Boehm

\begin{tabular}{|c|c|c|c|c|c|c|c|c|c|c|c|c|c|c|c|c|c|c|c|c|c|}
\hline \multirow{2}{*}{$\begin{array}{l}\text { Cuadro 3b } \\
\text { Ítems }\end{array}$} & \multicolumn{21}{|c|}{ Juegos tradicionales analizados, presentados en el Cuadro 1} \\
\hline & 21 & 22 & 23 & 24 & 25 & 26 & 27 & 28 & 29 & 30 & 31 & 32 & 33 & 34 & 35 & 36 & 37 & 38 & 39 & 40 & Total \\
\hline 1. Arriba & & & & & $\mathrm{X}$ & & & & & & & & & & & & & & & & 1 \\
\hline 2. A través & & & & & $\mathrm{X}$ & & & & & & & & & & & & & & & & 1 \\
\hline 3. Lejos & & & & & & & & & & & & & & & & & & & & & $\mathbf{0}$ \\
\hline 4. Junto a & & & & & & & & & & & & & & & & & & & & & $\mathbf{0}$ \\
\hline 5. Dentro & & & & & & & & & & & & & & & & & & & & & $\mathbf{0}$ \\
\hline $\begin{array}{l}\text { 6. Algunas, } \\
\text { pero pocas }\end{array}$ & & & $\mathrm{X}$ & & & $\mathrm{X}$ & & & & & & & & & & & & & & & 2 \\
\hline 7. Medio & & & & & & & & & & & & & & & & & & & & & 0 \\
\hline 8. Pocas & & & $\mathrm{X}$ & & & $\mathrm{X}$ & & & & & & & & & & & & & & & 2 \\
\hline 9. Más Lejos & & & & & & & & & & & & & & & & & & & & & $\mathbf{0}$ \\
\hline 10. Alrededor & & & & & & & & & $\mathrm{X}$ & & & & & $\mathrm{X}$ & & & & & & & 2 \\
\hline 11. Encima & & & & & & & & & & & & & & & & & & & & & 0 \\
\hline 12. Más ancha & & $X$ & & & & & & & & & & & & & & & & & & & 1 \\
\hline 13. Más & & & $\mathrm{X}$ & & & $\mathrm{X}$ & $\mathrm{X}$ & & $\mathrm{X}$ & & & & $\mathrm{X}$ & & & & $\mathrm{X}$ & & $\mathrm{X}$ & & 7 \\
\hline 14. Entre & & & & & & & & & $\mathrm{X}$ & & & & & $X$ & & & & & & & 2 \\
\hline 15. Entera & & & & & & & & & & & & & & & & & & & & & $\mathbf{0}$ \\
\hline 16. Más cerca & & $\mathrm{X}$ & & & & & & & & & & & & & & & & & & & 1 \\
\hline 17 Segundo & & & & $\mathrm{X}$ & & & & & & & & & & & $\mathrm{X}$ & & & & & $\mathrm{X}$ & 3 \\
\hline 18. Esquina & & & & $\mathrm{X}$ & & & & $\mathrm{X}$ & & & & & & & & & $\mathrm{X}$ & & & & 3 \\
\hline 19. Varios & & & $\mathrm{X}$ & & & $\mathrm{X}$ & & & & & & & & & & & & & & & 2 \\
\hline 20. Detrás & & & & & & & & & & & & & & & & & & & & & $\mathbf{0}$ \\
\hline 21. Fila & & & & $X$ & & & & & & & & & & & $X$ & & & & & $X$ & 3 \\
\hline 22. Diferente & & & & & & & $\mathrm{X}$ & & & & & & & & & & & & $\mathrm{X}$ & & 2 \\
\hline 23. Después & & & & $\mathrm{X}$ & & & & & & & & & & & $X$ & & & & & $\mathrm{X}$ & 3 \\
\hline 24. Casi & & & $\mathrm{X}$ & & & $\mathrm{X}$ & $\mathrm{X}$ & & $\mathrm{X}$ & & & & $\mathrm{X}$ & & & & $\mathrm{X}$ & & $\mathrm{X}$ & & 7 \\
\hline 25. Mitad & & & & & & & & & & & & & & & & & & & & & $\mathbf{0}$ \\
\hline 26. Centro & & & & $\mathrm{X}$ & & & & $\mathrm{X}$ & & & & & & & & & $\mathrm{X}$ & & & & 3 \\
\hline 27. Tantas & & & & & & & & & & & & & & & & & & & & & 0 \\
\hline 28. Lado & & & & $\mathrm{X}$ & & & & $\mathrm{X}$ & & & & & & & & & $\mathrm{X}$ & $\mathrm{X}$ & & & 4 \\
\hline 29. Empezando & & & & $\mathrm{X}$ & & & & & & & & & & & $\mathrm{X}$ & & & & & $\mathrm{X}$ & 3 \\
\hline 30. Otro & & & & & & & & $\mathrm{X}$ & & & & & & & & $\mathrm{X}$ & & & & & 2 \\
\hline 31. Semejante & & & & & & & & $\mathrm{X}$ & & & & & & & & $\mathrm{X}$ & & & & & 2 \\
\hline $\begin{array}{l}\text { 32.Ni primero, } \\
\text { ni último }\end{array}$ & & & & $\mathrm{X}$ & & & & & & & & & & & $\mathrm{X}$ & & & & & $\mathrm{X}$ & 3 \\
\hline 33. Nunca & & & $\mathrm{X}$ & & $\mathrm{X}$ & & & $X$ & & & & & & & & $\mathrm{X}$ & & $\mathrm{X}$ & & & 5 \\
\hline 34. Debajo & & & $\mathrm{X}$ & & & & $\mathrm{X}$ & & & & & & & $\mathrm{X}$ & & & & & & & 3 \\
\hline 35. Hace pareja & & & & $\mathrm{X}$ & & & & & & & & & & & $\mathrm{X}$ & & & & & $\mathrm{X}$ & 3 \\
\hline 36. Siempre & & & & & & & & $\mathrm{X}$ & & & & & & & & $\mathrm{X}$ & & & & & 2 \\
\hline $\begin{array}{l}\text { 37. Tamaño } \\
\text { mediano }\end{array}$ & & & & & & & & & & & & & & & & $\mathrm{X}$ & & $\mathrm{X}$ & & & 2 \\
\hline 38. Derecha & & & & & & & & $\mathrm{X}$ & & & & & & & & & & $\mathrm{X}$ & & & 2 \\
\hline 39. Adelante & & & & & & & & $\mathrm{X}$ & & & & & & & & & & $\mathrm{X}$ & & & 2 \\
\hline 40. Cero & $\mathrm{X}$ & $\mathrm{X}$ & $\mathrm{X}$ & $\mathrm{X}$ & & $\mathrm{X}$ & & & & $\mathrm{X}$ & & & $\mathrm{X}$ & & $\mathrm{X}$ & & $\mathrm{X}$ & & & $\mathrm{X}$ & 10 \\
\hline 41. Por encima & & & & & & & & & & & & & & & & & & & & & $\mathbf{0}$ \\
\hline 42. Cada & & & & $\mathrm{X}$ & & & & & & & & & & & $\mathrm{X}$ & & & & & $\mathrm{X}$ & 3 \\
\hline 43. Separadas & & & & & & & & & $\mathrm{X}$ & & & & & $\mathrm{X}$ & & & & & & & 2 \\
\hline 44. Izquierda & & & & & & & & $\mathrm{X}$ & & & & & & & & & & $\mathrm{X}$ & & & 2 \\
\hline 45. Par & & & & $\mathrm{X}$ & & & & & & & & & & & $\mathrm{X}$ & & & & & $\mathrm{X}$ & 3 \\
\hline 46. Saltarse & & & & $\mathrm{X}$ & & & & & & & & & & & $\mathrm{X}$ & & & & & $\mathrm{X}$ & 3 \\
\hline 47. Igual & & & & & & & & $\mathrm{X}$ & & & & & & & & $\mathrm{X}$ & & & & & 2 \\
\hline 48. En orden & & & $\mathrm{X}$ & & $\mathrm{X}$ & $\mathrm{X}$ & & & & & & & $\mathrm{X}$ & & & & & & & & 4 \\
\hline 49. Tercero & & & & $\mathrm{X}$ & & & & & & & & & & & $\mathrm{X}$ & & & & & $\mathrm{X}$ & 3 \\
\hline 50. Menos & & & & & & & & & & & & & & & & $\mathrm{X}$ & & $\mathrm{X}$ & & & 2 \\
\hline Total & 1 & 3 & 9 & 14 & 4 & 7 & 4 & 11 & 5 & 1 & 0 & $\overline{\mathbf{0}}$ & 4 & 4 & 11 & 7 & 6 & 7 & 3 & 11 & \\
\hline
\end{tabular}

Resultados del análisis de contenido. Fuente: Elaboración propia de las autoras 
Ejemplos de juegos del Catálogo elaborado, que se describió en el Cuadro 1:

Figura 5: Juego Damas chinas.

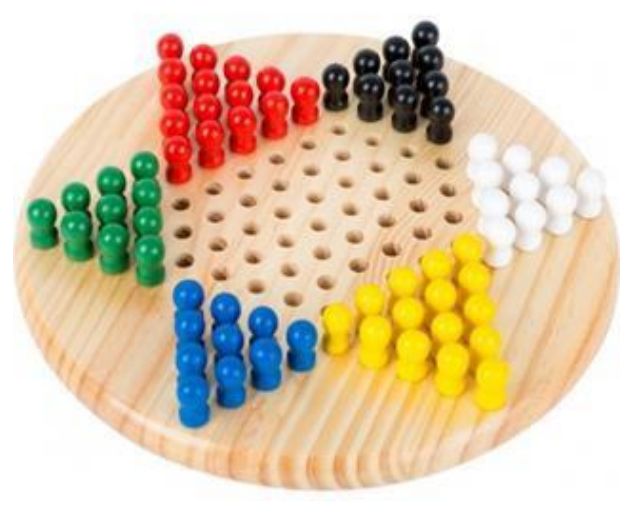

Fuente: Google imágenes

Figura 6: Juego Mijnlieff.

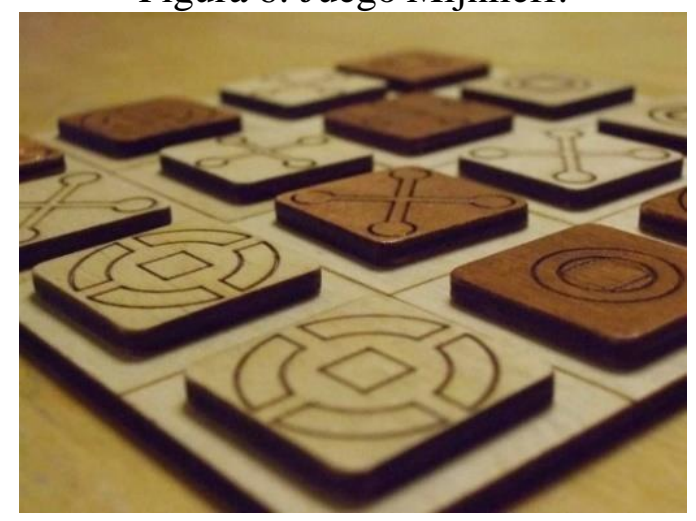

Fuente: Google imágenes

Figura 7: Juego Rayuela

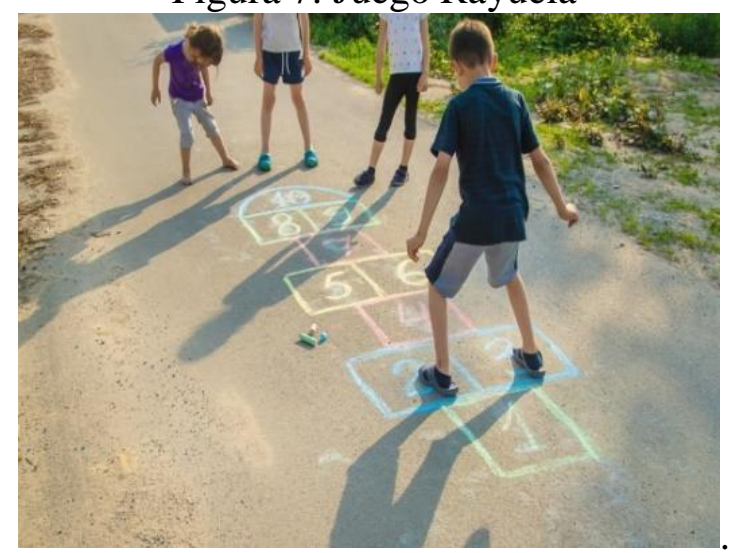

Fuente: Google imágenes

Los cuadros C 3a y C 3b, se sintetizan en el siguiente Cuadro 4, para realizar su análisis numérico: 
Cuadro 4: Cantidad de Juegos que evalúa cada ítem. Síntesis de los Cuadros C3a y C3b

\begin{tabular}{|c|c|c|c|}
\hline \multirow[b]{2}{*}{ Ítems test Boehm } & \multicolumn{3}{|c|}{ Cantidad de Juegos que evalúa cada ítem } \\
\hline & $\begin{array}{l}\text { Total } \\
\text { C3 a }\end{array}$ & $\begin{array}{l}\text { Total } \\
\text { C3 b }\end{array}$ & $\begin{array}{l}\text { Total de } \\
\text { Juegos }\end{array}$ \\
\hline 1. Arriba & 1 & 1 & 2 \\
\hline 2. A través & 1 & 1 & 2 \\
\hline 3. Lejos & 3 & $\mathbf{0}$ & 3 \\
\hline 4. Junto a & 3 & $\mathbf{0}$ & 3 \\
\hline 5. Dentro & 3 & $\mathbf{0}$ & 3 \\
\hline 6. Algunas, pero pocas & 7 & 2 & 9 \\
\hline 7. Medio & 1 & $\mathbf{0}$ & 1 \\
\hline 8. Pocas & 7 & 2 & 9 \\
\hline 9. Más Lejos & 3 & $\mathbf{0}$ & 3 \\
\hline 10. Alrededor & 1 & 2 & 3 \\
\hline 11. Encima & 3 & 0 & 3 \\
\hline 12. Más ancha & 3 & 1 & 4 \\
\hline 13. Más & 6 & 7 & 13 \\
\hline 14. Entre & 1 & 2 & 3 \\
\hline 15. Entera & 1 & $\mathbf{0}$ & 1 \\
\hline 16. Más cerca & 3 & 1 & 4 \\
\hline 17. Segundo. & 1 & 3 & 4 \\
\hline 18. Esquina & 2 & 3 & 5 \\
\hline 19. Varios & 7 & 2 & 9 \\
\hline 20. Detrás & 3 & $\mathbf{0}$ & 3 \\
\hline 21. Fila & 1 & 3 & 4 \\
\hline 22. Diferente & 2 & 2 & 4 \\
\hline 23. Después & 1 & 3 & 4 \\
\hline 24. Casi & 6 & 7 & 13 \\
\hline 25. Mitad & 1 & $\mathbf{0}$ & 1 \\
\hline 26. Centro & 2 & 3 & 5 \\
\hline 27. Tantas & 2 & $\mathbf{0}$ & 2 \\
\hline 28. Lado & 2 & 4 & 6 \\
\hline 29. Empezando & 1 & 3 & 4 \\
\hline 30. Otro & 2 & 2 & 4 \\
\hline 31. Semejante & 2 & 2 & 4 \\
\hline 32. Ni primero, ni último & 1 & 3 & 4 \\
\hline 33. Nunca & 3 & 5 & 8 \\
\hline 34. Debajo & 3 & 3 & 6 \\
\hline 35. Hace pareja & 1 & 3 & 4 \\
\hline 36. Siempre & 2 & 2 & 4 \\
\hline 37. Tamaño mediano & 2 & 2 & 4 \\
\hline 38. Derecha & 1 & 2 & 3 \\
\hline 39. Adelante & 4 & 2 & 6 \\
\hline 40. Cero & 11 & 10 & 21 \\
\hline 41. Por encima & 5 & $\mathbf{0}$ & 5 \\
\hline 42. Cada & 3 & 3 & 6 \\
\hline 43. Separadas & 1 & 2 & 3 \\
\hline 44. Izquierda & 1 & 2 & 3 \\
\hline 45. Par & 1 & 3 & 4 \\
\hline 46. Saltarse & 4 & 3 & 7 \\
\hline 47. Igual & 2 & 2 & 4 \\
\hline 48. En orden & 5 & 4 & 9 \\
\hline 49. Tercero & 1 & 3 & 4 \\
\hline 50. Menos & 2 & 2 & 4 \\
\hline
\end{tabular}

Fuente: Elaboración propia de las autoras 
En cuanto a los resultados obtenidos en el estudio, tras el análisis de los datos de C 3a, C 3 b y Cuadro 4, se aprecia que: 1) todos los ítems cuentan con al menos un juego que permite evaluarlo; 2) los juegos 6, 31 y 32 no conectan explícitamente con ningún ítem, sin embargo tienen en común la: Observación. Planificación y Resolución de problemas, por lo que se pueden utilizar para evaluar estas competencias, que son tanto matemáticas como científicas, y transversales a todos los ítems, como ya indicamos anteriormente.

La media de ítems que se manifiestan por cada juego es de 6, una cifra muy favorable para la investigación puesto que supone más de un ítems del test por juego, siendo 4 los juegos que tienen solo 1 ítem y 3 los juegos que contabilizando 0 ítem sin embargo representan las competencias metodológicas implícitas en todos los ítems. Mientras que la media de juegos por ítem es 4, sin tener en cuenta los tres casos de ítems atípicos con 13 o 21 juegos relacionados, lo que significa que se pueden evaluar todos los ítems con al menos un juego y la mayoría con varios juegos, lo que permitirá elegir el más afín con cada cultura, ya que tenemos cierta referencia a la cultura o zona geográfica en la que usa, en el cuadro 1.

Por otro lado, basándonos en los juegos como elementos que potencias capacidades matemáticas y científicas, hemos podido comprobar como los juegos con más potencial, ya que representan a 10 o más ítems son: 2 (20-20), 3 (Ajutatut), 7(Brown girl in the ring), 13 (Damas Chinas), 14 (Dandy Shandy), 24 (Hundir la flota), 28 (Molino de 9) ,35(Pass di ball) y 40 (ST-O-P). Se observa como de estos 9 juegos 5 son psicomotores y 4 son de tablero, algo que también nos parece interesante, puesto que indican que estos dos tipos de capacidades están presenten en juegos con alto PMC, y también se demuestra que las capacidades matemáticas se manifiestan jugando, independientemente de la naturaleza del juego. Lo cual, potencia el valor educativo del juego como elemento de aprendizaje y evaluación de competencias matemáticas y científicas en alumnado de Educación Infantil.

Para llevar a cabo la evaluación de los estudiantes con este instrumento hemos construido los elementos operativos del mismo, denominados: Protocolo de Observación, Ficha de Reflexión y Rúbrica de Evaluación, que también son resultados de la investigación y presentamos a continuación (Cuadros 5, 6, 7): 
Cuadro 5: Protocolo de Observación, válido para todos los juegos

\begin{tabular}{|l|l|l|l|l|}
\hline \multicolumn{5}{|c|}{ PROTOCOLO DE OBSERVACIÓN } \\
\hline $\begin{array}{l}\text { Atiende a las órdenes del } \\
\text { monitor sobre las normas } \\
\text { previas de organización } \\
\text { del juego }\end{array}$ & $\begin{array}{l}\text { Respeta su } \\
\text { turno en el } \\
\text { juego }\end{array}$ & $\begin{array}{l}\text { Respeta al compañero } \\
\text { o los compañeros de } \\
\text { juego relacionándose } \\
\text { correctamente }\end{array}$ & $\begin{array}{l}\text { Comprende las } \\
\text { reglas del } \\
\text { juego }\end{array}$ & $\begin{array}{l}\text { Termina el juego en el } \\
\text { tiempo indicado y } \\
\text { utilizando las reglas } \\
\text { adecuadamente }\end{array}$ \\
\hline
\end{tabular}

El Protocolo de observación, la ficha de reflexión y la Rúbrica, son instrumentos que hacen operativa la evaluación del dominio de cada contenido por el estudiante, y se han diseñado de forma que sean válidas para todos los juegos. Van dirigidas al evaluador o profesor y la Ficha es también para el alumno-jugador, que debe realizarla bajo la observación del evaluador, del mismo modo que cada juego desarrollado para evaluar. El protocolo guía las notas de campo, que realizará el evaluador mientras se desarrolla el juego, pudiendo recibir ayuda de un colaborador, o filmando en video el desarrollo de la actividad lúdica.

La ficha de reflexión (Cuadro 6) permite observar la internalización del concepto tratado en el juego e ítem y constituye a su vez la dimensión dos de la Rúbrica de evaluación del dominio del contenido de los ítems (dimensión 2: éxito en la representación del ítem mediante un dibujo del juego, elaborado con posterioridad a la realización del juego).

Cuadro 6: Ficha de reflexión, válida para todos los juegos

\begin{tabular}{|c|c|c|c|}
\hline \multicolumn{4}{|c|}{ FICHA DE REFLEXIÓN y representación de la vivencia experimentada al jugar } \\
\hline $\begin{array}{l}\text { Realizar un dibujo de la } \\
\text { actividad realizada en el } \\
\text { juego, incluyendo todos } \\
\text { los elementos del juego, } \\
\text { (Por ejemplo: pelota, } \\
\text { cancha de juego, canasta, } \\
\text { jugadores, etc.) }\end{array}$ & $\begin{array}{l}\text { (El espacio aconsejable } \\
\text { para esta ficha es la hoja } \\
\text { de papel habitual en las } \\
\text { tareas escolares, Din A4 } \\
\text { cuartilla o similar) }\end{array}$ & $\begin{array}{l}\text { Señalar en el dibujo la } \\
\text { situación o contenido } \\
\text { solicitado en el ítem, } \\
\text { (por ejemplo: la } \\
\text { pelota pasando "a } \\
\text { través de" la canasta) }\end{array}$ & $\begin{array}{l}\text { (Se puede hacer un ensayo } \\
\text { solicitando que señale el } \\
\text { elemento más importante del } \\
\text { dibujo y a continuación } \\
\text { solicitar que señale el } \\
\text { contenido del ítem) }\end{array}$ \\
\hline
\end{tabular}

Estos instrumentos de recogida de datos permiten evaluar los diferentes aspectos considerados relevantes para explicitar los niveles del éxito en el desarrollo del juego y del dominio de los conceptos de cada ítem, y especialmente pueden ser valorados directamente con la siguiente Rúbrica, que incluye dos dimensiones, siete criterios cualitativos y cuantificados y cinco niveles de éxito, explicitados de forma cualitativa (Cuadro 7). 
Cuadro 7: Rúbrica para la evaluación del dominio de los contenidos del test Boehm por jugadores de juegos con potencial matemático y científico PMC, válida para todos los juegos

\begin{tabular}{|c|c|c|c|c|c|}
\hline \multicolumn{6}{|c|}{ DIMENSIÓN 1: ÉXITO EN EL JUEGO } \\
\hline CRITERIOS & Muy baja (1-2) & Baja (3-4) & Media (5-6) & Alta (7-8) & Muy alta (9-10) \\
\hline $\begin{array}{l}\text { Atención a las órdenes del } \\
\text { monitor sobre las normas } \\
\text { previas de juego }\end{array}$ & $\begin{array}{l}\text { Hay que repetirle las normas } \\
\text { varias veces y no se interesa } \\
\text { por el juego }\end{array}$ & $\begin{array}{l}\text { Hay que repetirle las } \\
\text { normas dos veces y se } \\
\text { confunde, mostrando }\end{array}$ & $\begin{array}{l}\text { Hay que repetirle las } \\
\text { normas una vez y las sigue } \\
\text { con cierto desinterés }\end{array}$ & $\begin{array}{l}\text { Hay que repetirle las } \\
\text { normas una vez y las sigue, } \\
\text { sin destacar }\end{array}$ & $\begin{array}{l}\text { Hay que repetirle las normas } \\
\text { una vez y las comprende y } \\
\text { sigue perfectamente con }\end{array}$ \\
\hline \multicolumn{6}{|l|}{$15 \%$} \\
\hline $\begin{array}{l}\text { Respeta su turno en } \\
\text { el juego }\end{array}$ & No & Pocas veces & La mayor parte de las veces & Sí, salvo algún despiste & Siempre \\
\hline \multicolumn{6}{|l|}{$15 \%$} \\
\hline $\begin{array}{l}\text { Respeta al compañero o } \\
\text { los compañeros de }\end{array}$ & No & Pocas veces & La mayor parte de las veces & Sí, salvo algún despiste & Siempre \\
\hline \multicolumn{6}{|l|}{$15 \%$} \\
\hline $\begin{array}{l}\text { Comprende las reglas } \\
\text { del juego }\end{array}$ & $\begin{array}{l}\text { Hay que repetirle las } \\
\text { reglas varias veces y } \\
\text { no las comprende }\end{array}$ & $\begin{array}{l}\text { Hay que repetirle la } \\
\text { explicación de las } \\
\text { reglas dos veces y las } \\
\text { siomea }\end{array}$ & $\begin{array}{l}\text { Hay que repetirle la } \\
\text { explicación de las reglas } \\
\text { una vez y las sigue a } \\
\text { medias con }\end{array}$ & $\begin{array}{l}\text { Hay que repetirle la } \\
\text { explicación de las } \\
\text { reglas una vez y las } \\
\text { sione con }\end{array}$ & $\begin{array}{l}\text { Hay que repetirle la } \\
\text { explicación de las reglas } \\
\text { una vez y las sigue, sin } \\
\text { ningún }\end{array}$ \\
\hline \multicolumn{6}{|c|}{ _medias con } \\
\hline $\begin{array}{l}\text { Termina el juego en el } \\
\text { tiempo indicado y } \\
\text { logrando el objetivo }\end{array}$ & $\begin{array}{l}\text { No logra el objetivo en } \\
\text { el tiempo indicado y } \\
\text { deja el juego a medias, } \\
\text { sin terminar }\end{array}$ & $\begin{array}{l}\text { No logra el objetivo en el } \\
\text { tiempo indicado y lo } \\
\text { termina en un tiempo } \\
\text { mayor, pero sin lograr el }\end{array}$ & $\begin{array}{l}\text { En el tiempo indicado lo } \\
\text { termina aunque sin lograr } \\
\text { el objetivo completamente }\end{array}$ & $\begin{array}{l}\text { Logra el objetivo en } \\
\text { un tiempo algo } \\
\text { mayor que el } \\
\text { indicado y lo termina }\end{array}$ & $\begin{array}{l}\text { Logra el objetivo en el } \\
\text { tiempo indicado y lo termina } \\
\text { correctamente }\end{array}$ \\
\hline \multicolumn{6}{|l|}{$15 \%$} \\
\hline \multicolumn{6}{|c|}{ DIMENSIÓN 2: ÉXITO EN LA REPRESENTACIÓN DEL ÍTEM MEDIANTE UN DIBUJO DEL JUEGO, REALIZADO CON POSTERIORIDAD A JUGARLO } \\
\hline CRITERIOS & Muy baja (1-2) & Baja (3-4) & Media (5-6) & Alta (7-8) & Muy alta (9-10) \\
\hline $\begin{array}{l}\text { Realiza un dibujo de } \\
\text { su actividad en el } \\
\text { juego, incluyendo } \\
\text { todos los elementos del }\end{array}$ & No realiza el dibujo & $\begin{array}{l}\text { Sí realiza el dibujo } \\
\text { pero incompleto }\end{array}$ & $\begin{array}{l}\text { Sí realiza el dibujo conla } \\
\text { mayoría de elementos } \\
\text { implicados, pero no } \\
\text { completo } \\
\end{array}$ & $\begin{array}{l}\text { Sí realiza el dibujo contodos } \\
\text { los elementos implicados, pero } \\
\text { falta él como jugador para estar } \\
\text { completo }\end{array}$ & $\begin{array}{l}\text { Sí realiza el dibujo con todos } \\
\text { los elementos implicados, y él } \\
\text { comojugador, todo completo }\end{array}$ \\
\hline \multicolumn{6}{|l|}{$12,5 \%$} \\
\hline $\begin{array}{l}\text { Realiza un dibujo de su } \\
\text { actividad en el juego y } \\
\text { señala en el la situación }\end{array}$ & No & $\begin{array}{l}\text { No señala lo indicado en } \\
\text { el ítem sino cualquier cosa }\end{array}$ & $\begin{array}{l}\text { Señala lo solicitado pero } \\
\text { de forma inadecuada }\end{array}$ & $\begin{array}{l}\text { Señala lo solicitado } \\
\text { de forma adecuada }\end{array}$ & $\begin{array}{l}\text { Señala lo solicitado } \\
\text { de forma adecuada y } \\
\text { lo explica }\end{array}$ \\
\hline \multicolumn{6}{|l|}{$12,5 \%$} \\
\hline Calificación Dimensión 1 & & Calificación Dimensión 2 & & Calificación Final & \\
\hline
\end{tabular}


La calificación final del alumno-jugador en cada ítem se puede contrastar con el índice de dificultad del ítem, obtenido en Oliveras (1984) y mostrado en la Tabla 1.

Sería deseable elaborar una evaluación cualitativa que indique las competencias más afianzadas y las menos o las inexistentes y redactar un perfil matemático-científico del niño, a tener en cuenta por el docente que trabaje con él en el primer curso de Primaria, para encauzar su aprendizaje de una forma personalizada, a la par que grupal, que le permita no fracasar en estas áreas, en las que el fracaso inicial propicia el abandono del aprecio a estas materias y las dificultades de aprendizaje en el futuro escolar.

Todo ello es posible mediante este instrumento de evaluación, que cumple con creces el objetivo del estudio planteado desde un inicio: elaborar un instrumento que permita evaluar las competencias matemáticas del alumnado que inicia Educación Primaria, mediante el test Boehm y los juegos tradicionales que hemos estudiado, pudiendo elegir aquellos que estén a su alcance en el centro escolar o en centros de educación no formal como las Ludotecas y talleres del entorno social o familiar.

\section{Referencias}

Barba-Martín, R. A., y López-Pastor, V. M. (2017). Evaluación formativa y compartida en los proyectos de trabajo tutorado, un ejemplo de buena práctica. Revista Infancia, Educación y Aprendizaje, 3 (2), pp.66-70.

Bergen, D. (2009). Play as the Learning Medium for Future Scientists,Mathematicians, and Engineers. American Journal of Play, 1 (4), pp. 413-428.

Boehm, A. (1971). Boehm Test of Basic Concepts. New York: The Psychological Corporation. Boehm, A. (1980). Test Boehm de Conceptos Básicos. Madrid: TEA.

Boehm, A. E. (2012). Boehm-3. Test Boehm de Conceptos Básicos - 3. Madrid: Pearson.

Brassard, MR y Boehm, AE (2007). Evaluación preescolar: principios y prácticas. Guilford Press.

Corbalán, F. (1997). Juegos de estrategia y resolución de problemas: Análisis de estrategias y tipología de jugadores en el alumnado de secundaria. (Tesis doctoral). Universitat Autònoma de Barcelona. Bellaterra (Barcelona).

Craft, A. (2008). Creativity in the School. London: UK Department for Children, Schools and Families' Beyond Current Horizons Project

Chang, C. P. (2013). Relationships between playfulness and creativity among students gifted in mathematics and science. Creative Education, 4(02), p.101.

De Castro Hernández, Carlos (2007) La evaluación de métodos para la enseñanza y el aprendizaje de las matemáticas en la Educación Infantil. Revista Iberoamericana de Educación Matemática (11). pp. 59-77. ISSN 1815-0640 (2018) 
De la Cruz, V. (1988): Pruebas de Diagnóstico de Preescolar. Madrid, TEA.

Delgado, I. (2011). ¿A qué jugamos? Los juegos, clasificación y funciones. En: El juego infantil y su metodología, (pp. 158-159). Madrid. España: Paraninfo.

Delors, J. (1996.): Los cuatro pilares de la educación, en: La educación encierra un tesoro. Informe a la UNESCO de la Comisión internacional sobre la educación para el siglo XXI, Madrid, España: Santillana/UNESCO. pp. 91-103.

Deulofeu, J. (2001). Una recreación matemática: historias, en el alumnado de secundaria. (Tesis doctoral). Universitat Autònoma de Barcelona. Bellaterra (Barcelona).

Espigares-Gámez, M. J. (2018). Juegos tradicionales jamaicanos como potenciadores del conocimiento matemático-científico en Educación Infantil y Primaria. (Trabajo fin de Máster). Universidad de Granada.

Espigares-Gámez, M. J., Fernández-Oliveras, A., y Oliveras, M. L. (2019). Compilation of tradicional games played in Jamaica. An ethnomathematical study for STEAM education. ICERI 2019.

Espigares-Gámez, M.-J., Fernández-Oliveras, A., y Oliveras, M.-L. (2019). Análisis de juegos. Catálogo de juegos tradicionales para trabajar áreas científicas y matemáticas. En: Sola, T; García; Fuentes, A; Rodríguez-García, A.M. y Belmonte, J. Innovación Educativa en la Sociedad Digital (pp. 2186-2200). Granada. Dykinson.

Evans, M.A. (2009). Mobility, Games and Education. In R.E. Ferdig (ed.), Handbook of Research on Effective Electronic Gaming in Education New York: Information Science Reference (pp. 96-110).

Fernández-Oliveras A y Oliveras M.L (2015). Formación de maestros y Microproyectos curriculares, Revista Latinoamericana de Etnomatemática, 8 (2), pp. 472-495.

Fernández-Oliveras, A., Espigares-Gámez, M. J., y Oliveras, M. L. (2019). Gamification and game-based learning for the development of STEAM skills in kindergarten, primary and secondary education. Updating initial teacher training. Simposio llevado a cabo en el seminario Innovation in Learning and teaching in Science, Technology, Engineering and Mathematics (STEM) fields. COIMBRA. Granada.

Fernández-Oliveras, A., Espigares-Gámez, M.-J., y Oliveras, M.-L. (2019). Teorizaciones para la tipificación de juegos con potencial educativo STEAM. En: Sola, T; García; Fuentes, A; Rodríguez-García, A.M y Belmonte, J. Innovación Educativa en la Sociedad Digital: Vol.

VII. Innovación e investigación educativa (pp. 1645-1658). Dykinson.

Gallardo, P. y Fernández, J. (2010). El juego como recurso didáctico en educación física. Sevilla: Wanceulen.

García Fraile, J. A. y Tobón, S. (2009). Estrategias didácticas para la formación por competencias. Lima: A. B. Representaciones Generales 
Garrison, C. y M. Ehringhaus (2008), Formative and Summative Assessments in the Classroom, tomado de http://www.nmsa.org/Default.aspx, página web de la National Middle School Association

Gil, F. (1999). Marco conceptual y creencias de los profesores sobre evaluación en matemáticas. (Tesis doctoral). Granada, España: Universidad de Granada.

Glenberg, A. M. y Robertson, D. A. (1999). Indexical understanding of instructions. Discourse Processes, 28 (1), pp.1-26. Doi :10.1080/ 01638539909545067.

Gómez, A. (2007), La evaluación en actividades de aprendizaje con uso de tecnología. ( Tesis de maestría con especialidad en Matemática Educativa), Cicata-IPN, México

Gómez, J. F. (2012). El juego infantil y su importancia en el desarrollo. CCAP, 10 (4), 5-13.

González González, C. S. (2015). Estrategias para trabajar la creatividad en la Educación Superior: pensamiento de diseño, aprendizaje basado en juegos y en proyectos. Revista de Educación a Distancia, 40. Recuperado a partir de https://revistas.um.es/red/article/view/234291.

Gros, B. (2000). La dimensión socioeducativa de los videojuegos. Edutec. Revista Electrónica de Tecnología Educativa, 12, pp.1-11. Recuperado de:

http://edutec.rediris.es/Revelec2/Revelec12/ gros.pdf

Harms,T.y Clifford ,D. (1980). Early Childhood Environment Rating Scale. New York, Teachers College Press.

Kangas, M. (2010). Creative and playful learning: Learning through game co-creation and games in a playful learning environment. Thinking skills and Creativity, 5 (1), pp.1-15.

Lera-Rodríguez, M.J (1994): Las ideas de los profesores y su práctica educativa, un estudio en preescolar. (Unpublished Tesis Doctoral), Universidad de Sevilla. Sevilla.

Lera-Rodríguez, M.J. (2007). Calidad de la Educación Infantil: instrumentos de evaluación.

Revista de Educación, 343, pp.301-323.

Morris, B., Croker, S., Zimmerman, C., Gill, D., y Romig, C. (2013). Gaming science: the "Gamification" of scientific thinking. Frontiers in psychology, 4, p.607.

Muñoz, A y Díaz M.R. (2009): Metodología por proyectos en el área de conocimiento del medio. Revista Docencia e Investigación, 19, pp.101- 126.

Narváez, A. (1987). Prueba de Conceptos Básicos de Boehm: Estudio Comparativo en niños de diferente nivel socioeconómico de Lima y Callao. (Tesis de Bachiller). PUC. Lima.

National Advisory Committee on Creative and Cultural Education (1999). All Our Futures: Creativity, Culture and Education. Report to the Secretary of State for Education and Employment the Secretary of State for Culture, Media and Sport, UK

National Council of Teachers of Mathematics (2000). Principles and standards for school mathematics. Reston, Va.: The National Council of Teachers of Mathematics

Navarro Guzmán, J. I., Aguilar Villagrán, M., Marchena Consejero, E., Alcalde Cuevas, C., y García Gallardo, J. (2010). Evaluación del conocimiento matemático temprano en una muestra de $3^{\circ}$ de Educación Infantil. Revista de Educación, 352, pp. 601-615 
Oliveras, M. L. (1984). Dominio del área de Matemáticas en niños de preescolar de Granada. Escuela de maestros 1, (pp. 115-124). Universidad de Granada.

Oliveras, M. L. (1996). Etnomatemáticas. Formación de profesores e innovación curricular. Granada: Comares

Oliveras, M. L. (2005). Microproyectos para la educación intercultural en Europa. Uno: Revista de Didáctica de las Matemáticas, 38 (11), pp.70-81.

Oliveras, M. L. (2006). Etnomatemáticas de la multiculturalidad al mestizaje. En: Matemáticas e interculturalidad. pp. 117-149. Biblioteca de UNO, Número 232. Barcelona: Graó.

Paredes, J. (2003). Juego, luego soy. Teoría de la actividad lúdica. Sevilla: Wanceulen Perrenoud, P. (2008). Construir las competencias, ¿es darle la espalda a los saberes? Revista de docencia universitaria, 6(2), pp.2-8. Núm. monograf. II www.redu.um.es/Red_U/m2

Piaget, J. (1973). Psicología y Pedagogía. Ciudad de la Habana: Editorial Pueblo y Educación.

Powers, B. (1986). Reliability of the Boehm Test of Basic Concepts for hispanic and non hispanic kindergarten pupils. Psychology in the Schools, 23, pp.34-36.

Resnick, M. (2004). Edutainment? No thanks. I prefer playful learning. Associazione Civita Report on Edutainment, 14, pp.1-4.

Rosas, R, Ceric, F, Aparicio, A, Arango, P, Arroyo, R, Benavente, C, Escobar, P, Olguín, P, Pizarro, M, Ramírez, M.P, Tenorio, M, y Véliz, S. (2015). Traditional Assessment or Invisible Assessment Using Games? New Frontiers in Cognitive Assessment. Psykhe (Santiago), 24 (1), pp. (s/p). Versión On-line ISSN 0718-2228. http://dx.doi.org/10.7764/psykhe.23.2.724.

Ruiz, L. (2005). Aprendizaje y matemáticas. La construcción del conocimiento matemático en la Escuela Infantil. Madrid: Pearson.

Tobón, S. (2006). Aspectos básicos de la formación basada en competencias. Proyecto Mesesup, 1, pp.1-16. Talca: Mesesup.

Toro, V. (2013). El juego como herramienta educativa del Educador Social en actividades de Animación Sociocultural y de Ocio y Tiempo libre con niños con Discapacidad. Revista de educación social, 16. pp.1-13

Vázquez-Alonso, A., Manassero-Mas, M. A. (2017). Juegos para enseñar la naturaleza del conocimiento científico y tecnológico. Educar, 53(1), pp.149-170.

Zhao, Z. y Linaza, J.L. (2015). La importancia de los videojuegos en el aprendizaje y el desarrollo de niños de temprana edad. Electronic Journal of Research in Educational Psychology, 13(2), pp.301-318. Doi: 10.14204/ejrep 


\section{Webgrafía.}

(La numeración corresponde con la de cada juego del Catálogo presentado en el Cuadro 1)

1. ¿Qué hora es señor lobo? Juegos Educación Física. [JuegosEF]. (2015.Enero.12) QUÉ HORA ES SEÑOR LOBO?-Juegos Educación Física [Archivo de vídeo]. Recuperado de https://www.youtube.com/watch?v=36pzVxoLZI

2. 20-20. Kamii. C (1989). 20-20 Baraja Matemtática. Madrid. Diario Educación. https://diarioeducacion.com/20-20-baraja-matematica/

3. Ajutatut. Santana., D [David Santana]. (2013, Noviembre 27). DIAPO11.1 [Archivo de vídeo] Recuperado de https://www.youtube.com/watch?v=5u3fDh_Gc3o

4. Awale. Matemáticas [iißMATE-MATE-MÁTICAS!!!] (2017, Octubre 24). ¡MATEMATE- MÁTICAS!! PRESENTA: EL AWALÉ. [Archivo de vídeo] Recuperado de https://www.youtube.com/watch?v=t6dyDIe-Ihk

5. Beggar my neighbourg. [Royds2oscn] (2013, Octubre 2) How to play Beggar my Neighbour [Archivo de vídeo] https://www.youtube.com/watch?v=iMTMXRaICvE

6. Bluebird. Distefano, D [Danielle Distefano] (2016, Febrero 4) Here Comes Bluebird GAME (Elem Methods) Danielle Distefano. [Archivo de vídeo] Recuperado de https://www.youtube.com/watch?v=c9iD4xthe2k

7. Brown Girl in the Ring. [JAFSProyect] (2018, Diciembre 15) Brown Girl in the Ring [Archivo de vídeo] Recuperado de https://www.youtube.com/watch?v=7Rg5XfLJLx0

8. Bruck Rock Stone [LearnVision10] (2012, Febrero,23). Go down a Enmanuel Road [Archivo de vídeo] Recuperado de : https://www.youtube.com/watch?v=YDYcNVGwrAg

9. Bull inna pen. Bull inna pen (s.f) Tafisa Recall. http://recallgames.com/games/90

10. Buzz Fizz. Hartley ,M. (s.f) Dr Mike's Math Games for Kids. http://www.dr-mikes-mathgames-for-kids.com/fizz-buzz.html

11. Shut the box. [Wilder Math Adventures] (2017, Septiembre 27) How to play Shut the Box [Archivo de vídeo] Recuperado de https://www.youtube.com/watch?v=aiYUFUbc3wE

12. Chinesse skip. [Eze Congco Dancehall Grung] (2018, Abril 1) Chiny/Chinese Skip ( Game) [Archivo de vídeo] Recuperado de https://www.youtube.com/watch?v=Zn-bFFx73Bw

13. Damas Chinas. Poma, D [David Poma Huanca] (2014) Damas Chinas [Archivo de vídeo] Recuperado de https://www.youtube.com/watch?v=1s8VCq2Z5NE

14. Dandy Shandy. Mckenzie, P [Patrick Mckenzie] (2011, Agosto 22 ) Dandy Shandy Glen Island Park [Archivo de vídeo] Recuperado de https://www.youtube.com/watch?v=iR2rubdJOho

15. Daruma Otoshi. [JapanToy Mania] (2014, Julio 17 ) Daruma Otoshi! Stacked Daruma Game! だるま落としゲーム [Archivo de vídeo] Recuperado de https://www.youtube.com/watch?v=G0UbuQJ4mNQ

16. Doga n’bone. [Blooming Kids Madhira] (20118, Diciembre 11) Dog and bone game [Archivo de vídeo] Recuperado de https://www.youtube.com/watch?v=u48KVRegAKg

17. Dominó. [JamDomDotCom] (2017, Mayo 17) How to Play French Dominoes- Jam Dom. Com [Archivo de vídeo] Recuperado de https://www.youtube.com/watch?v=u48KVRegAKg

18. Fanorona.[Rincón Lúdco] (2016, Spetiembre 17). Fanororna [Archivo de vídeo] Recuperado de https://www.youtube.com/watch?v=vd0-6Zbr9QE

19. Farmers in the dell. Dozet, GN [George N. Dozet] (2009) The Farmer in the Dell [Archivo de vídeo] Recuperado de https://www.youtube.com/watch?v=mibD51R4Kco 
20. Follow the arrow. (s.f) BBG. https://boardgamegeek.com/boardgame/126530/followarrow

21. Gig Spinning. Juego tradicional. El trompo (2017). Guía Infantil.com. https://www.guiainfantil.com/articulos/educacion/juegos/juego-tradicional-de-lapeonza-el-trompo/

22. Hide n'seek. Juego del escondite (s.f) Con mis hijos. https://www.conmishijos.com/ninos/ocio/juego-del-escondite-juegos-tradicionales- paraninos/

23 y 37. Hopscatch. Juego de la Rayuela. (s.f) Con mis hijos. https://www.conmishijos.com/ ninos/ocio/juego-de-la-rayuela-como-jugar-con-los- ninos/

24. Hundir la flota. [juguetes review] (2019, Julio 12) BATTLESHIP JUEGO como jugar/ juguetes review [Archivo de vídeo] Recuperado de https://www.youtube.com/watch?v=IR6MFrq50-M

25. La Mamba. Cuentanos África (s.f) http://cuentanosafrica.blogspot.com/2014/01/juegos_12.html

26. Loodi. Anderson, T [Mr Talique Anderson] (2018, Julio 22) How To Play LOODI (Caribbean Game) [Archivo de vídeo] Recuperado de https://www.youtube.com/watch?v=46Te9Hyk1 y4

27. Marbles. [Howcast] (2017) How to play Marbles [Archivo de vídeo] Recuperado de https://www.youtube.com/watch?v=ewqFhNw9k7g

28. Mijnlieff. [Exacting Games] (2018, Febrero 4 ) Mijnlieff: Gameplay ( Standard Board Setup)- EG [Archivo de vídeo] Recuperado de https://www.youtube.com/watch?v=610gRd5Mh98

29. Molino de 9. [La Jirafa de Madera] (2019, Julio 27) ¿Cómo jugar Molino? ( 9 hombres Morris) [La Jirafa de Madera] [Archivo de vídeo] Recuperado de https://www.youtube.com/watch?v=eX0QE1HBo2g

30. Morra. [El juego de la Morra] (2017, Agosto 7) La partida de morra más multitudinaria de la historia (68 jugadores) [Arxhivo de vídeo] Recuperado de https://www.youtube.com/watch?v=ZdKRNxhIb_s

31 y 32 Mummy Lashy. Clapping games ( s.f). https://www.wgtn.ac.nz/lals/research/projects/ language-in-the-playground- project/publications/lip70.pdf

33. Pajaritos. Juego 31. (s.f) https://www.juegossolitario.com/juego/Treinta+y+uno+\%2831\%29

34 y 35 Pass di ball / Pasa el aro. Classroom games. (2019) https://games4esl.com/pass-the$\underline{\text { ball/ }}$

36. Pass round donkey (Burro). https://en.wikipedia.org/wiki/Donkey_(card_game)

38. River bank game. [Teachers Hub] (2018, Diciembre 21) River Bank / Party Games / Classroom Games / Fun games [Archivo de vídeo] Recuperado de https://www.youtube.com/watch?v=Xk9K1jGeL0g

39. Saca Yunya. El juego andino. (2008). https://es.slideshare.net/mediadora/el-juego-en-elnio-andino

40. S-T-O-P. Busy Teacher. ( s.f) https://busyteacher.org/25070-stop-game.html 